\title{
Behavioral and Circuit Basis of Sucrose Rejection by Drosophila Females in a Simple Decision-Making Task
}

\author{
CDChung-Hui Yang, ${ }^{1}$ Ruo He, ${ }^{1}$ and Ulrich Stern ${ }^{2}$ \\ ${ }^{1}$ Department of Neurobiology, Duke University Medical School, Durham, North Carolina 27710 and ${ }^{2}$ Yang Laboratory, Durham, North Carolina 27705
}

\begin{abstract}
Drosophila melanogaster egg-laying site selection offers a genetic model to study a simple form of value-based decision. We have previously shown that Drosophila females consistently reject a sucrose-containing substrate and choose a plain (sucrose-free) substrate for egg laying in our sucrose versus plain decision assay. However, either substrate is accepted when it is the sole option. Here we describe the neural mechanism that underlies females' sucrose rejection in our sucrose versus plain assay. First, we demonstrate that females explored the sucrose substrate frequently before most egg-laying events, suggesting that they actively suppress laying eggs on the sucrose substrate as opposed to avoiding visits to it. Second, we show that activating a specific subset of DA neurons triggered a preference for laying eggs on the sucrose substrate over the plain one, suggesting that activating these DA neurons can increase the value of the sucrose substrate for egg laying. Third, we demonstrate that neither ablating nor inhibiting the mushroom body (MB), a known Drosophila learning and decision center, affected females' egg-laying preferences in our sucrose versus plain assay, suggesting that MB does not mediate this specific decision-making task. We propose that the value of a sucrose substrate - as an egg-laying option — can be adjusted by the activities of a specific DA circuit. Once the sucrose substrate is determined to be the lesser valued option, females execute their decision to reject this inferior substrate not by stopping their visits to it, but by actively suppressing their egg-laying motor program during their visits.
\end{abstract}

Key words: decision making; dopamine; Drosophila; egg laying; sucrose rejection

\section{Introduction}

The ability to choose "the greater of two goods" is advantageous for animal survival. To do so, animals must assess and rank the values of their choice options. Primate studies have made significant progress in elucidating the neural basis of "goods-based decisions" (Wallis, 2007; Glimcher et al., 2009; Padoa-Schioppa, 2011). In particular, activities of some neurons in the orbital frontal cortex have been shown to correlate with the values of different food options (Tremblay and Schultz, 1999; PadoaSchioppa and Assad, 2006, 2008; Padoa-Schioppa, 2013). However, the exact mechanisms by which animal brains transform sensory stimuli associated with choice options into values, rank the values, and translate the result of ranking into decision outcomes remain incompletely understood.

Drosophila egg-laying site selection offers a genetically tractable system to study the neural basis of a simple value-based deci-

Received March 12, 2014; revised Nov. 23, 2014; accepted Nov. 26, 2014.

Author contributions: C.-H.Y. and U.S. designed research;C.-H.Y., R.H., and U.S. performed research;C.-H.Y., R.H., and U.S. analyzed data; C.-H.Y. and U.S. wrote the paper.

C.-H.Y. is funded by a Klingenstein Award, a Whitehead Award, and the National Institutes of Health under award number R01GM100027. We thank Drs. F. Wolf, D. Anderson, M. Wu, B. Dickson, K. Scott, P. Garrity, T. Lee, and the Bloomington Stock Center for providing us with fly strains. We also thank the Duke Physics Shop, especially Phil Lewis, for helping us build various behavioral apparatus. We also thank Drs. B. Ye, L. Glickfeld, S. Lisberger, and members of the Yang lab for critically reading this manuscript.

The authors declare no competing financial interests.

Correspondence should be addressed to Chung-Hui Yang, Department of Neurobiology, Duke University Medical School, Durham, NC 27710. E-mail: yang@neuro.duke.edu., (919) 684-1494.

DOI:10.1523/JNEUROSCI.0992-14.2015

Copyright $\odot 2015$ the authors $\quad 0270-6474 / 15 / 351396-15 \$ 15.00 / 0$ sion. Several studies have shown that Drosophila are highly selective about where to lay their eggs and can withhold egg laying when there is no acceptable substrate available (Yang et al., 2008; Joseph et al., 2009; Joseph and Heberlein, 2012; Schwartz et al., 2012; Azanchi et al., 2013; Dweck et al., 2013). More importantly, Drosophila can rank their options and reject "the lesser of two goods" (Yang et al., 2008; Azanchi et al., 2013). For example, when choosing egg-laying sites in a specific two-choice assay we designed, females readily accept a sucrose substrate when it is the sole option, but reject it when a sucrose-free option is also available (Yang et al., 2008). This observation suggests that while females consider both options acceptable, they value the sucrose substrate less in our assay. The mechanism by which females determine the values of these substrates and execute their decision to reject the lesser valued sucrose substrate is unknown.

Here we investigate the mechanism that underlies females' rejection of the sucrose substrate in our sucrose versus plain assay, using new tools we developed. Behavioral tracking showed that females explored the sucrose substrate before most egg-laying events-perhaps to feed. Analysis of the duration and timing of these explorations revealed that females rejected the sucrose substrate not by avoiding it but by suppressing egg laying on it. Circuit manipulation showed that activating a set of little-described DA neurons triggered a preference for laying eggs on the sucrose-containing substrates, suggesting that activating these neurons increases the value of the sucrose substrates. Moreover, neither inhibiting nor ablat- 
ing the mushroom body (MB), a known Drosophila learning and decision center (Zhang et al., 2007; Joseph et al., 2009; Azanchi et al., 2013; DasGupta et al., 2014), changed how females chose between the sucrose versus plain options, suggesting the MB does not mediate this decision. Our results suggest that, when making the sucrose versus plain decision in our assay, females consider the sucrose substrate the inferior choice, and execute their decision by suppressing their egg-laying motor program when visiting it. Activating a specific DA circuit elevates the value of the sucrose substrate, allowing females the flexibility to turn it into the superior choice when appropriate.

\section{Materials and Methods}

\section{Fly husbandry}

Flies were raised at either room temperature or $25^{\circ} \mathrm{C}$ and with the humidity set at $60 \%$. We used the standard cornmeal molasses food to maintain and raise the flies (but we used different substrates to test their egg-laying preferences, see next section). In addition, we supplied wet yeast paste ( $6 \mathrm{~g}$ of active yeast mixed with $10 \mathrm{ml}$ of $0.5 \%$ propionic acid) to food vials to ensure that flies were well fed for several days before being transferred into our apparatus to be tested for their egg-laying preferences. The following stocks were used: W1118, CS, TH-GAL4 (FriggiGrelin et al., 2003); Ddc(HL5)-GAL4 (Kong et al., 2010); C'-GAL4 (Q. Liu et al., 2012); UAS-mCD8-GFP (BL\# 29715); UAS-dTRPA1 (Hamada et al., 2008); DR $1^{\text {attP }}$ (Keleman et al., 2012); DR $1^{f 02676}, D 2 R^{f 06521}$, $D R 2^{M B 05108}, D R 2^{a t t P}$ (Keleman et al., 2012); DopEcR $R^{C 02142}$, DopE$c R^{M 102790}, 247-L e x A$, LexAop-shi ${ }^{T S}$ (Lin et al., 2014); and $\Delta E P(X)-5$; $G r 64 a^{2} / G r 64 a^{2}$ (aka $\Delta G r 5 a ; \Delta G r 64 a$; Dahanukar et al., 2007).

\section{Egg-laying preference assays}

Content of our egg-laying substrates. All of our egg-laying substrates were made from agarose. The contents of our substrates were as follows: (1) plain contained included $1 \%(\mathrm{w} / \mathrm{v})$ agarose, (2) sucrose substrate contained $1 \%$ agarose with $150 \mathrm{~mm}$ sucrose, (3) juice substrate contained 1\% agarose with $3 \times$ diluted store-bought juices, (4) firm substrate contained $1.8 \%$ agarose, and (5) firm and sweet $\left(\right.$ Firm $\left._{\text {sweet }}\right)$ substrate contained $1.8 \%$ agarose with $150 \mathrm{~mm}$ sucrose.

Preparation of our egg-laying substrates. We routinely kept bottles of 1 and $1.8 \%$ agarose preheated in a $55^{\circ} \mathrm{C}$ water bath (to maintain the agarose in liquid form). To prepare the sucrose substrate, for example, the sucrose stock solution $(2 \mathrm{M}$ ) was preheated in a $50 \mathrm{ml}$ conical tube for $\sim 5 \mathrm{~min}$ in the same water bath. We then added the preheated agarose to the tube to yield a final concentration of $150 \mathrm{~mm}$ sucrose, mixed by vortexing, and then dispensed the substrates into each individual trough in our egg-laying apparatus. Plain substrate was prepared in the same manner but preheated $\mathrm{H}_{2} \mathrm{O}$ was added to the preheated agarose instead.

Preparation of flies to be assayed. Thirty-five to 40 virgins of the appropriate genotypes and $\sim 25$ males of mixed genotypes were collected into a single food vial that was supplied with wet yeast paste. These flies were left in the vials for $\sim 5 \mathrm{~d}$ until the food in the vial became very chewed up by the larvae. At this point, females were well fed but deprived of egg laying because the food was too soft and wet for them to lay eggs on. Thus, they were ready to lay eggs when placed in our high-throughput egg-laying apparatus. This method is more convenient than the one we originally described (Yang et al., 2008).

Assembling the egg-laying preference apparatus. When the flies were ready to be assayed, we loaded them into the top portion of our apparatus and let them recover from $\mathrm{CO}_{2}$ for at least $30 \mathrm{~min}$. We then started preparing the bottom plates where the egg-laying substrates were placed. In our 30-chamber apparatus, there were 12 troughs, and each held $\sim 950 \mu \mathrm{l}$ of agarose. After we loaded the substrates into these troughs, we let them air dry for 30 min before assembling the top and bottom portions of the apparatus. Once assembled, the entire apparatus was either placed at room temperature or $32^{\circ} \mathrm{C}$ depending on the experimental conditions required. We usually let females lay eggs overnight $(\sim 14 \mathrm{~h})$.
Behavioral analysis. To track the positions of females, we mounted four webcams (Microsoft LifeCam Cinema) on top of the egg-laying apparatus using a custom-built holder. Females and chambers were prepared as described earlier but we recorded behaviors of egg-laying females for $8 \mathrm{~h}$ only. We used CamUniversal software for video acquisition, Avidemux software for video conversion, and the open-source tracking software Ctrax (Branson et al., 2009) for tracking. Individual egg-laying events in the videos were manually annotated. We routinely scored five videos ( $\sim 250$ egg-laying events) for each genotype. Each $8 \mathrm{~h}$ video took $\sim 1 \mathrm{~h}$ to annotate. Ctrax was used to convert exploratory behaviors of females into trajectories. To analyze the Ctrax-generated trajectories, we used custom MATLAB and Python code (available upon request).

\section{$M B$ ablation with hydroxyurea}

To ablate the MB, we collected larvae that were hatched within $1 \mathrm{~h}$ and placed them in inactive yeast paste mixed with hydroxyurea (HU; SigmaAldrich) for $5 \mathrm{~h}$. The treated larvae were then removed and raised on standard food. For controls, we followed the same procedure except that the inactive yeast paste was not mixed with $\mathrm{HU}$.

\section{Immunohistochemistry}

For FasII staining, we followed the standard fixation and staining protocol. For TH staining, however, brains were dissected, permeabilized, and fixed using a specific protocol (Mao and Davis, 2009). The anti-FasII monoclonal antibody was obtained from the Developmental Studies Hybridoma Bank. The anti-mCD8 antibody was obtained from Molecular Probes. The anti-TH antibody was from Millipore. Alexa 488- and RRXconjugated secondary antibodies were from Jackson ImmunoResearch. After staining and washing following a standard protocol, samples were mounted in SlowFade Antifade reagent (Invitrogen), and images were acquired using a Zeiss LSM 700 confocal.

\section{Results}

Drosophila females rejected the sucrose substrate when selecting between the sucrose versus plain substrates for egg laying in our high-throughput apparatus

To begin to understand the neural basis of Drosophila females' rejection of the sucrose substrate for egg laying in our sucrose versus plain assay (Yang et al., 2008), we first improved the throughput of our assay. We constructed several highthroughput apparatuses, each of which can assay egg-laying preferences of 30 individual animals simultaneously (Fig. 1A). Importantly, our new apparatus also enables speedy preparation of egg-laying substrates, contains individual chambers that are larger than the ones we had previously used (so females have more roaming space), and uses a plastic divider to prevent diffusion between the two agarose-based substrates (Fig. 1B).

Females readily laid eggs in the new apparatus: a single, well fed wild-type female could deposit over 50 eggs overnight when provided, for example, with $1 \%$ agarose substrates that contained grape juice, or pineapple juice, or $150 \mathrm{~mm}$ sucrose (Fig. 1C; ANOVA: $F_{(2,39)}=0.06, p=0.94$; Fig. $1 E$ ). Also, in keeping with our earlier findings, while females readily laid eggs on the sucrose substrate when it was the sole option, they robustly rejected the sucrose substrate when a plain option was available (Fig. 1D; Yang et al., 2008). Moreover, females' rejection of a sugary substrate-when it was pitted against a plain one-persisted when we replaced the sucrose substrate with grape or pineapple juice substrates (Fig. 1D). This result suggests that females' decision to reject the sugary substrate over a plain one for egg laying in our assay can be generalized to sweet substances that can be found in nature.

Because the plain substrate and the sucrose substrate in our assay differed only in their sucrose content (1\% agarose versus $1 \%$ agarose with $150 \mathrm{~mm}$ sucrose), we next assessed whether it was the chemosensory quality of sucrose that caused females to reject the sucrose 
substrate. We tested the egg-laying preferences of mutant females that lack two known sucrose-sensing gustatory receptors, Gr5a and Gr64a ( $\Delta G r 5 a ; \Delta G r 64 a$; Dahanukar et al., 2007), and found that they no longer rejected the sucrose substrate for egg laying (ANOVA: $F_{(3,115)}=40.34, p<$ 0.0001 ; Fig. $1 F$ ). This result suggests detection of sucrose by females' sweet taste receptors is responsible for triggering the rejection of the sucrose substrate in our assay.

These results demonstrated the effectiveness of our new high-throughput apparatus. Moreover, they confirmed our previous finding that females readily accept a sucrose substrate when it is presented alone but reject it when it is presented together with a plain substrate (Yang et al., 2008), suggesting that females consider the sucrose substrate an acceptable but inferior option for egg laying in our sucrose versus plain decision task.

\section{The system we devised to record} females and plot their trajectories as they explored and laid eggs in our decision chambers

We next began investigating the behavioral strategies Drosophila females employ to execute their decisions to reject the sucrose substrate for egg laying. Because females do lay eggs on a sucrose substrate when exploring it in other contexts (Yang et al., 2008; Schwartz et al., 2012), we hypothesized that they must adjust their behavioral response to the sucrose substrate when choosing an egg-laying site in our sucrose versus plain chambers (to not lay eggs on it). To test this idea, we first developed a system that allowed us to record the behaviors of individual females as they laid eggs and explored the egg-laying substrates for $8 \mathrm{~h}$ (Fig. 2A,B). After each recording, we processed the videos with several additional steps. First, we manually annotated all the egg-laying events in each video. Similar to our earlier observation (Yang et al., 2008), we found that females typically did not start laying eggs until at least $20 \mathrm{~min}$ after being placed into the chambers (Fig. 2C). Once they started to lay eggs, they laid one at a time and did so in a temporally random manner (i.e., there was no fixed time interval in between two consecutive egg-laying events; Fig. 2C). In the second postprocessing step, we used the open-source tracking software Ctrax (Branson et al., 2009) to track the flies, yielding the females' positions over time, which, in turn, allowed us to plot trajectories with egg-laying events annotated in them (Fig. 2D). Last, we used our custom MATLAB and Python code to assess females' substrate exploration pattern before each egg-laying event.

E
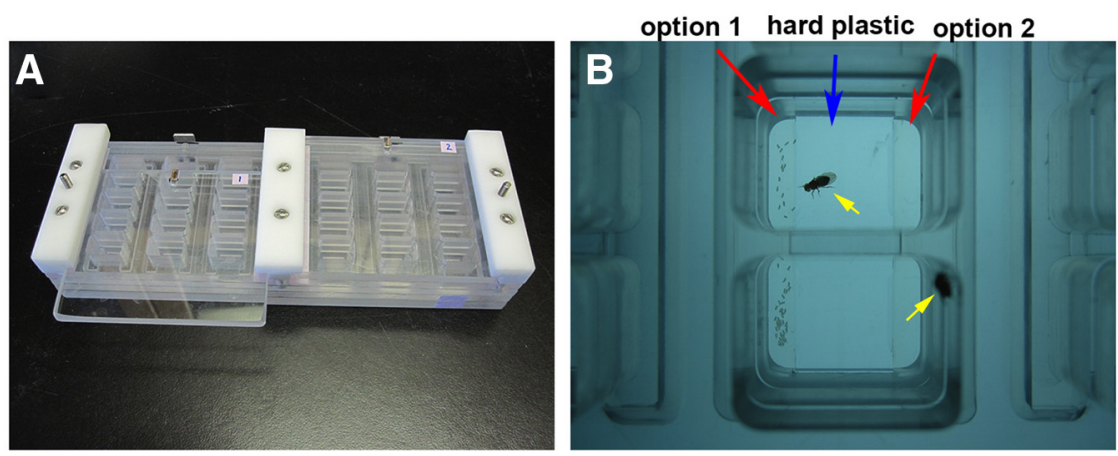

S: $1 \%$ agarose $+150 \mathrm{mM}$ sucrose

G: $1 \%$ agarose $+3 \mathrm{X}$-diluted grape juice

$\mathrm{Pa}: 1 \%$ agarose $+3 \mathrm{X}$-diluted pineapple juice
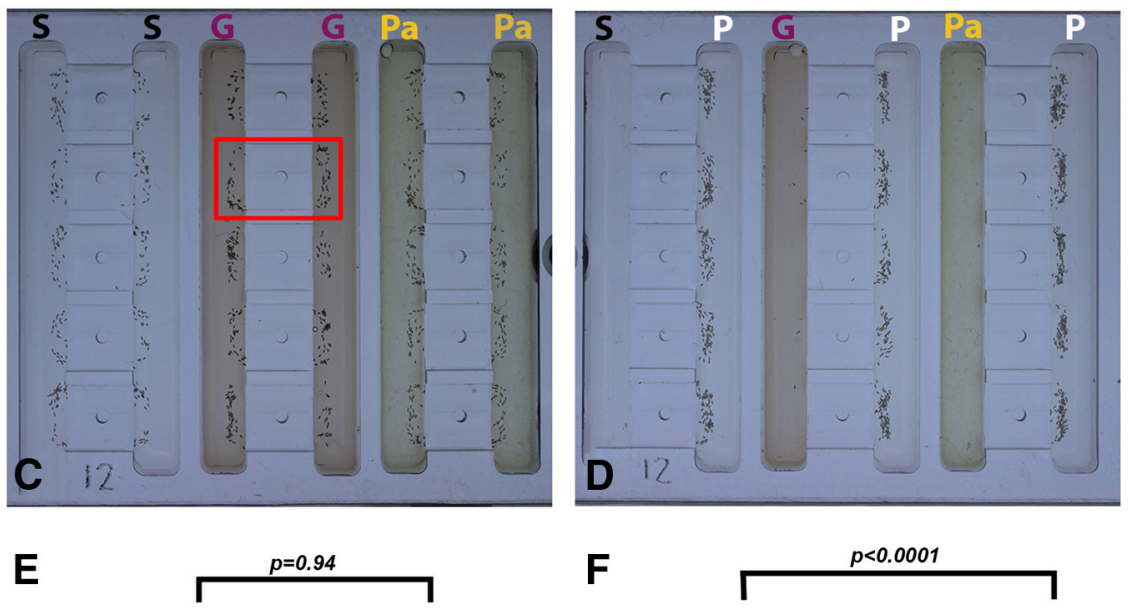

$\mathbf{F}$

$p<0.0001$
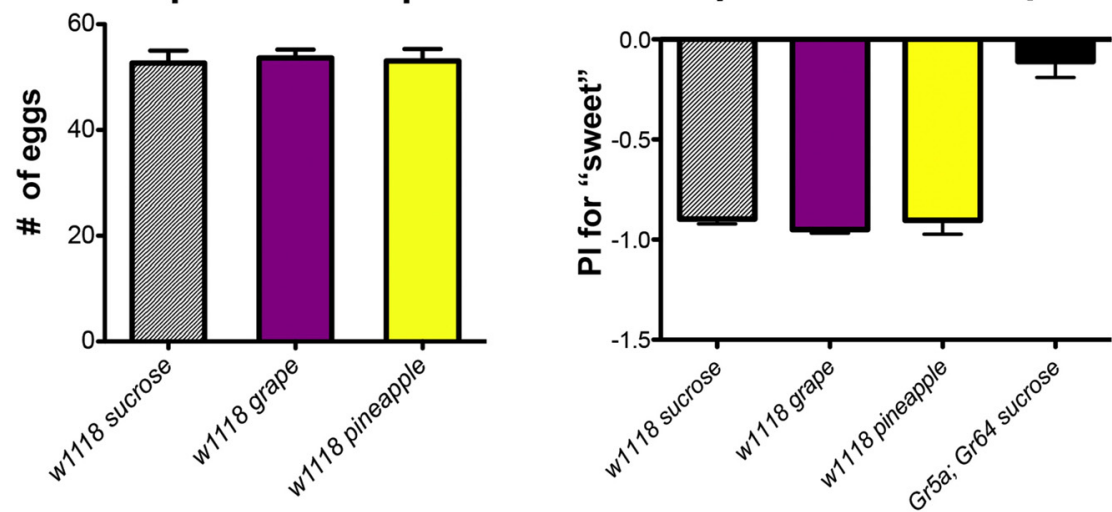

Figure 1. Drosophila females preferred laying eggs on the nonsweet substrates over the sweet ones in our high-throughput apparatus. $\boldsymbol{A}$, Shows the egg-laying apparatus we designed. $\boldsymbol{B}$, Picture showing two individual chambers, each containing a single egg-laying female (yellow arrow). Each chamber has two troughs for placing egg-laying substrates. $\boldsymbol{C}$, Females readily laid eggs on the sucrose $(S)$, grape juice $(G)$, and pineapple juice $(\mathrm{Pa})$ substrates when they were the sole option. The red rectangle denotes the approximate area covered by a single chamber. The dark specks are eggs. $D$, Females preferred to lay their eggs on the plain substrate $(P)$ when choosing between a sweet and a plain substrate. $\boldsymbol{E}$, Quantification of the numbers of eggs laid by single animals assayed as shown in $C . N=30$ for each group. Note that all data shown in this work are mean \pm SEM. The statistical tests and the $p$ values are described in the main text. $\boldsymbol{F}$, Quantification of the Preference Index (PI) of single females assayed as shown in D. PI for each animal was calculated as follows: (\# of eggs on the sweet site - \# of eggs on the plain site)/(\# of eggs on both sites). A positive PI indicates a preference for laying eggs on the sweet substrate, and a negative Pl indicates a preference for the plain substrate. Wild-type flies showed a preference for the plain substrate but $\Delta G r 5 a ; \Delta G r 64 a$ mutants showed no preference. $N=30$ for each group.

\section{Analysis of females' behavioral response toward the sucrose} option when they were laying eggs in our sucrose versus plain decision chambers

To begin to assess how females modify their behavior toward the sucrose substrate in our sucrose versus plain chambers, we examined the occurrence, timing, and duration of their su- 

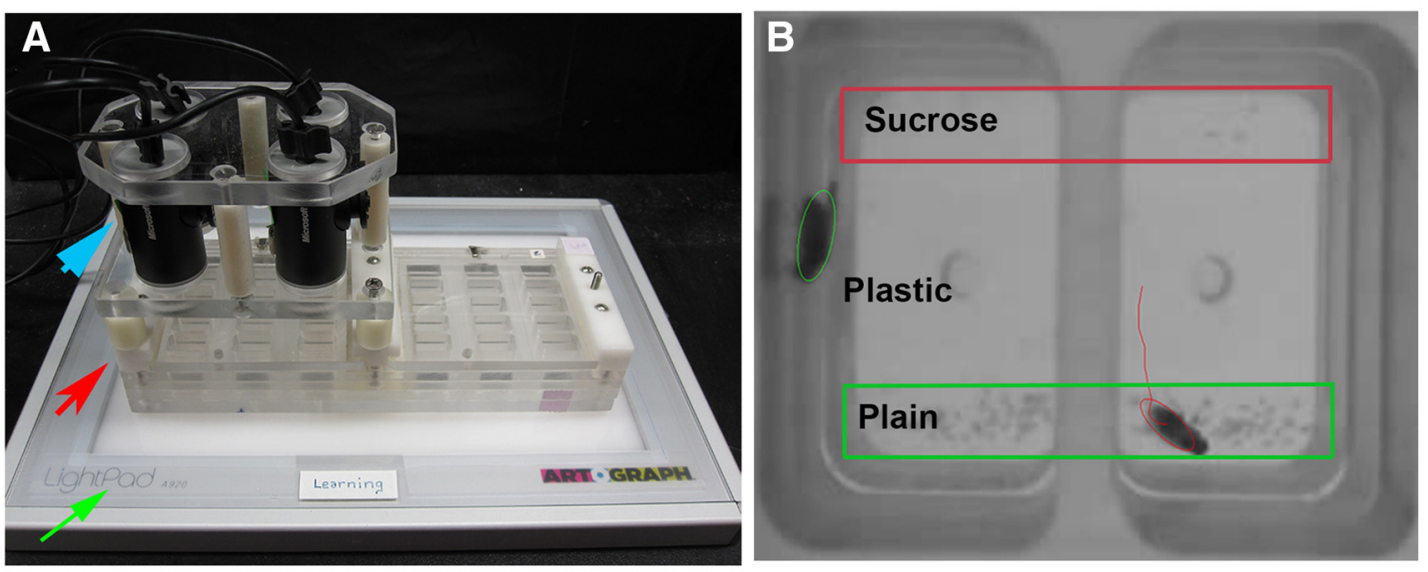

C

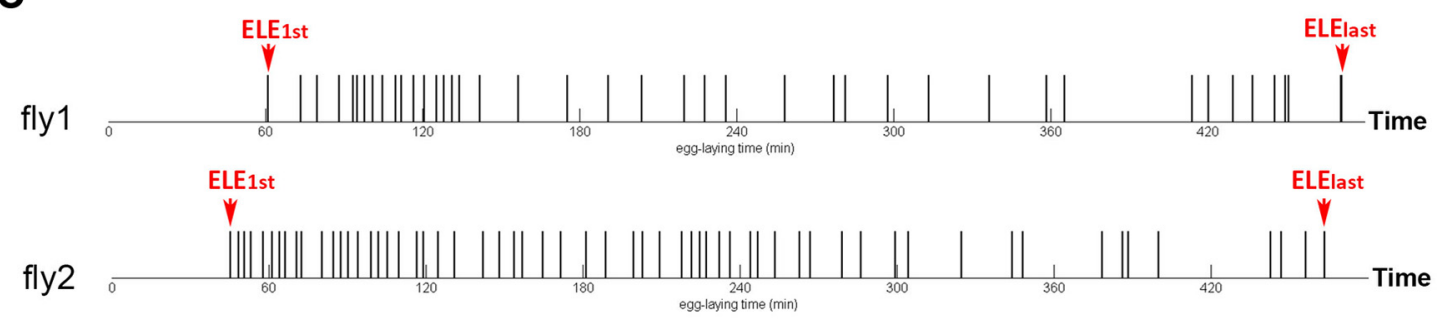

D

V1 -. Fly 2, Period 2

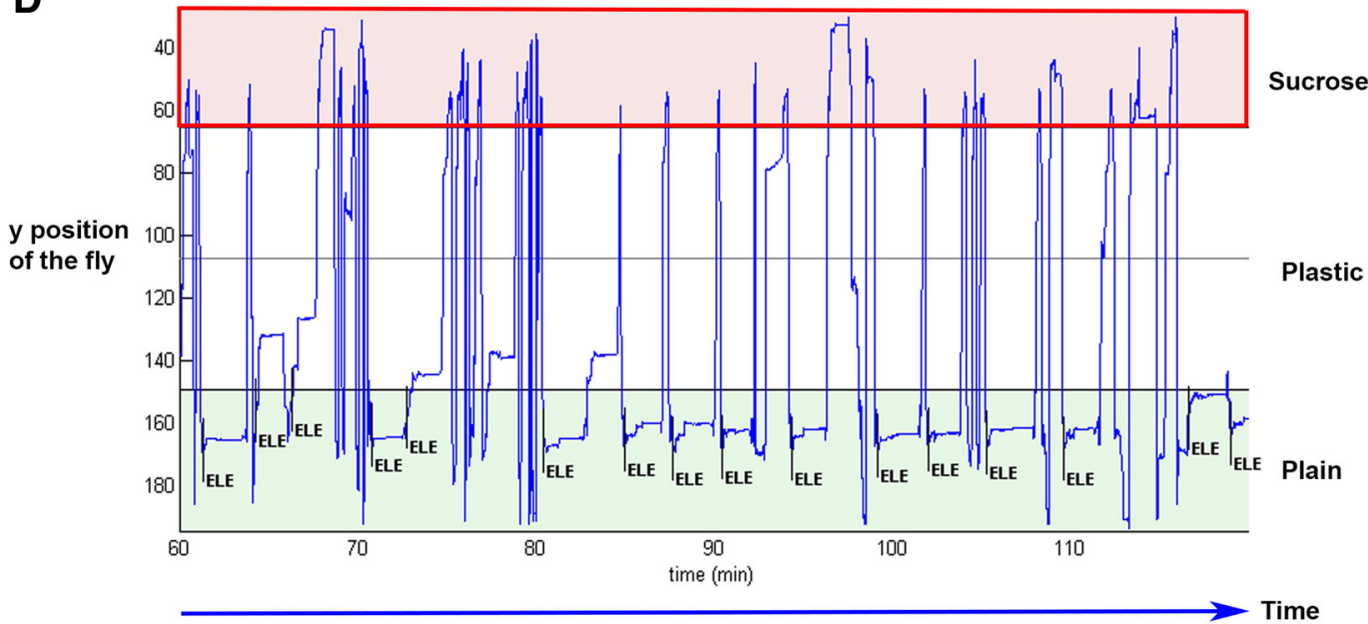

Figure 2. Our system to record and plot trajectories of egg-laying females. $A$, Shows the recording setup we designed. Four cameras (blue arrow) were mounted on top of the egg-laying apparatus (red arrow). The light pad (green arrow) provided illumination. Each camera can record two females in two chambers. B, Picture showing two females being tracked by Ctrax. C, Egg-laying time lines of two females. Long tick mark, egg-laying event; $\mathrm{ELE}_{\text {first' }}$ the first egg-laying event; $\mathrm{ELE}_{\text {last' }}$ the last egg-laying event. Note that both females laid their first egg only after exploring the chamber for $>30 \mathrm{~min}$. D, Trajectory with the time of each ELE annotated in it. Blue trace, y position over time.

crose visits during a specific time window before each egglaying event (ELE). We termed this specific window "the oneback period" (Fig. $3 A$ ). It begins at the previous egg-laying event $\left(\mathrm{ELE}_{\mathrm{n}-1}\right)$ and terminates at the egg-laying event of interest $\left(\operatorname{ELE}_{\mathrm{n}}\right)$. Because this is the window when females are getting ready to deposit the next egg $\left(\mathrm{ELE}_{\mathrm{n}}\right)$, observing how they behave toward the sucrose substrate in this window should inform us about the behavioral strategy they employ to reject the sucrose substrate for egg laying. For example, we can envision at least four possible strategies. First, once egg laying commences (i.e., after $\mathrm{ELE}_{\text {first }}$ ), females stop visiting the sucrose substrate. Second, females visit the sucrose substrate only when they just finished laying an egg (so that they do not yet have another egg ready for deposition). Third, they make their sucrose visits too brief to allow the egg-laying motor program to be activated. Fourth, they actively suppress the activation of their egg-laying motor program during their sucrose visits. In the following sections, we examined each possibility by analyzing females' sucrose visits.

Possibility I: females no longer visited the sucrose substrate before most ELEs

We first assessed how often females visited the sucrose substrate during the one-back period before each ELE. We found that for $89 \%$ of the ELEs we annotated (206 of 232), females visited the sucrose substrate at least once during the one-back period. This is evident in the example shown in Figure 3B: the one-back period before each of the 23 listed ELEs contains at least one sucrose visit. 

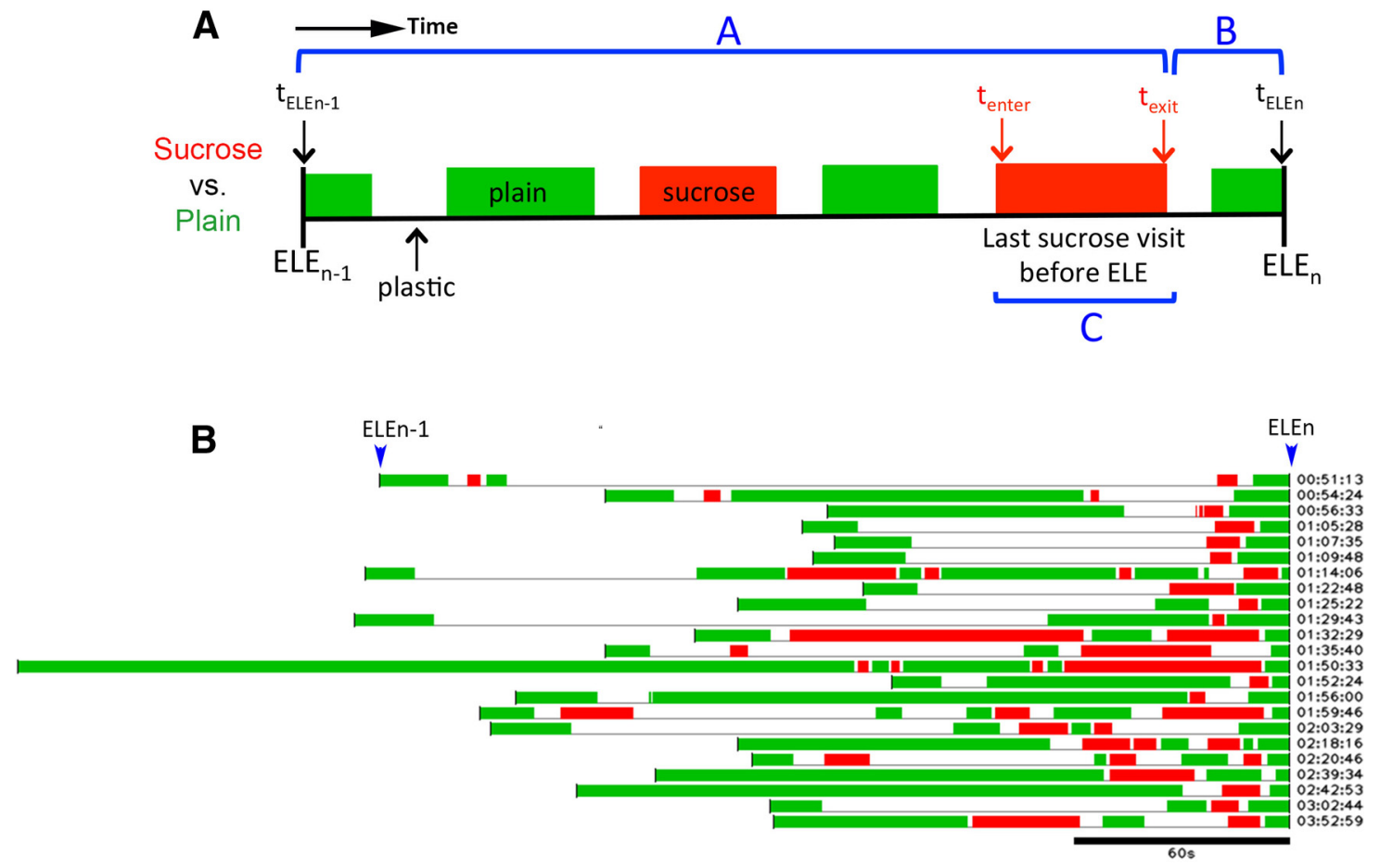

C

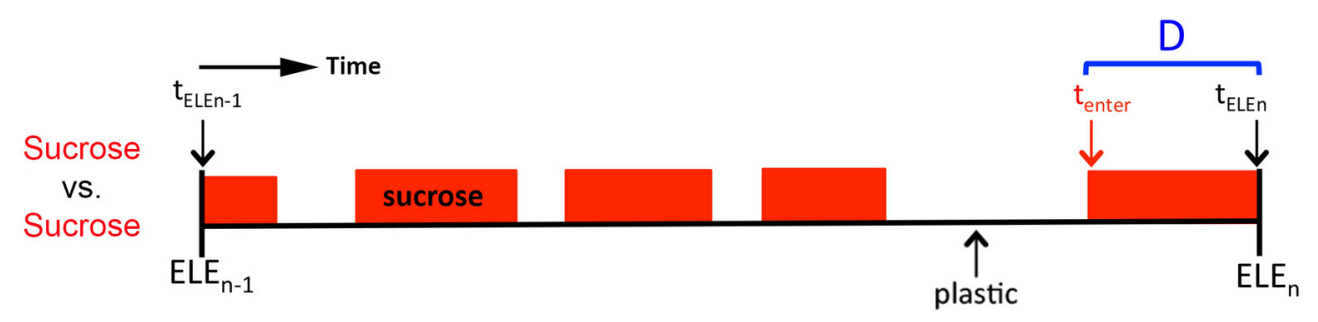

D

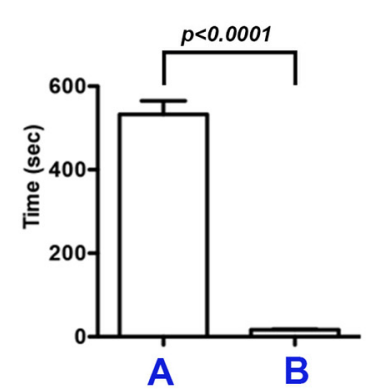

E

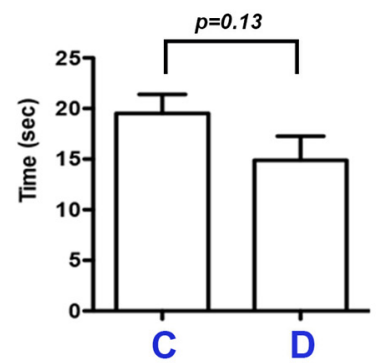

Figure 3. Females visited the sucrose substrate before most egg-laying events in the sucroseversus plain chambers, and thesevisits should haveallowed egg laying. $A$, Diagram depicting the one-back period for an $E L E\left(E L E_{n}\right)$. One-back period is defined as the time window that begins at the time of the prior egg-laying event $\left(E_{E} E_{n-1}\right)$ and ends at the time of the egg-laying event of interest ( $\left(E L E_{n}\right)$. During this window, females may visit both substrates. A green block indicates a visit to the plain substrate, a red block a visit to the sucrose substrate, and an "empty space" time spent on plastic. $t_{E L E n-1}$ and $t_{E L E n}$, $t_{\text {ime of }} E_{L E} E_{n-1}$ and

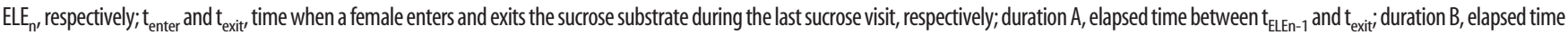
between $\mathrm{t}_{\text {exit }}$ and $\mathrm{t}_{\mathrm{ELE}}$; ;uration C, elapsed time for the last sucrose visit. Females may pay several visits to the sucrose substrate during a one-back period; the visit that occurs the closest to $\mathrm{ELE}_{\mathrm{n}}$ is defined as the last sucrosevisit. $B$, Female's substrate exploration patterns during the one-back periods before $23 E L E s$. The time of each ELEis labeled on the right. C, Diagram depicting the one-back period forthesucroseversus

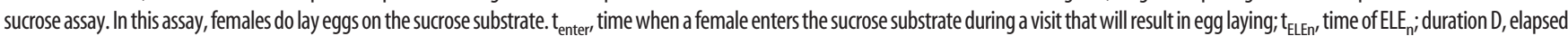
time between $t_{\text {enter }}$ until $t_{\mathrm{ELEn},}$, which reflects the time of contact with the sucrose substrate females need to activate the egg-laying motor program to deposit an egg onto it. $\boldsymbol{D}$, The time when a female exited the sucrose substrate during the last sucrose visit was closer to the time of $\mathrm{ELE}_{n}$ than $\mathrm{ELE}_{\mathrm{n}-1}$ in the sucrose versus plain assay. Columns $\mathrm{A}$ and $\mathrm{B}$; see also diagram in panel $\boldsymbol{A} . \mathbf{N}=227,207$, respectively. $\boldsymbol{E}, \mathrm{A}$ comparison of the amount of time a female spent on the last sucrose visit in the sucrose versus plain assay (column C) to the time of contact with the sucrose substrate a female needs to activate the egg-laying motor program (column D). $N=209$ for column C, $N=184$ for column D.

Therefore, females regularly explored the sucrose substrate before most egg-laying events, arguing against full positional avoidance of the sucrose substrate as their means to reject laying eggs on the sucrose substrate in our sucrose versus plain decision chambers.
Possibility II: females restricted their sucrose visits to only immediately after an ELE

It takes time to for an egg to descend from the ovaries to reach the uterus (where only one egg can be held and fertilized at a time). Thus females are physically unready to lay another egg for a short 
period of time after each egg-laying event. We therefore wondered whether females might prevent themselves from laying eggs on the sucrose substrate by restricting their sucrose visits to only when they just finished laying an egg. To test this idea, we examined the time when females terminated their "last sucrose visit" (Fig. $3 A$, duration $C$ ). If such a visit, on average, terminated close to the time when females just finished laying an egg (Fig. $3 A$, $\left.t_{\text {ELEn-1 }}\right)$, the females may have been unready to lay eggs on sucrose, but if such visit terminated close to the time of the upcoming egg-laying event (Fig. $3 A$, $\mathrm{t}_{\mathrm{ELEn}}$ ), the females should have been ready to lay eggs on sucrose. We found that, on average, females' last sucrose visit terminated much closer to the time of the upcoming ELE ( $t$ test: $t_{(227)}=15.91, p<0.0001$; Fig. $\left.3 D\right)$, suggesting that females do not prevent themselves from laying eggs on the sucrose substrate by restricting their sucrose visits to only when they just finished laying an egg.

Possibility III: duration of females' last sucrose visit was too brief to support activation of the egg-laying motor program

We have noticed that females always physically explore a potential substrate for a short period of time before initiating the egglaying motor program to deposit an egg onto it (Yang et al., 2008; the egg-laying motor program consists of females inserting their ovipositor into the egg-laying substrates and contracting their abdominal muscles to expel an egg out of the uterus). Our observation therefore suggests that females need sufficient time of contact with a substrate before they are able to activate their egg-laying motor program to deposit an egg onto the substrate. Thus, females might refrain from laying eggs on the sucrose substrate by paying only brief visits to the sucrose substrate, thereby not allowing themselves sufficient time to initiate the egg-laying motor program. To test this idea, we first determined the time of contact with the sucrose substrate females need to lay eggs on it. We did this by calculating the time elapsed between when females entered the sucrose substrate (Fig. $3 C, \mathrm{t}_{\text {enter }}$ ) and when they deposited an egg onto it (Fig. $3 C$, $\mathrm{t}_{\mathrm{ELEn}}$ ) in the sucrose versus sucrose assay where females do lay eggs on the sucrose substrate. We next determined the amount of time females typically spent on their last sucrose visit (Fig. 3A, duration $\mathrm{C}$ ) in the sucrose versus plain assay. We found that the duration of their last sucrose visit was comparable to the time of contact with sucrose substrate they needed to activate the egg-laying motor program $\left(t\right.$ test: $t_{(359)}=$ $1.42, p=0.13$; Fig. $3 E$ ). Thus, this result suggests females do not reject the sucrose substrate by reducing the length of their sucrose visits.

Together, our analysis of females' behavior in many oneback periods revealed that females almost always visited the sucrose substrate before executing each egg-laying decision. Moreover, these sucrose visits should, in principle, support egg laying on the sucrose substrate: their duration met the length requirement for activating the egg-laying motor program, and they tended to occur when females should be physically ready to lay another egg. Therefore, our results suggest that when deciding where to lay eggs in our sucrose versus plain assay, females most likely actively suppressed the activation of their egg-laying motor program during their sucrose visits, once they had learned that a more valuable plain option was also available. When such learning took place is unclear, however. It could have happened during the first $20 \mathrm{~min}$ after females were introduced into the choice chamber. Alternatively, females might have relearned the quality of the options offered on an ongoing basis.
Activating TH-expressing neurons resulted in females' preferring to lay eggs on the sucrose substrate in our sucrose versus plain decision chambers

Our results so far suggest that Drosophila females consider the sucrose substrate the lesser option in our sucrose versus plain assay, and that they execute their decision to reject it by actively suppressing egg laying on it. But how do females decode the values of their egg-laying substrates? To answer this question, we began by searching for neurons that, when activated, can alter females' egg-laying preferences. Specifically, we used the GAL4UAS system (Brand and Perrimon, 1993) to express in candidate neurons the heat-gated cation channel dTRPA1, an effective and widely used tool for activating neurons in Drosophila (Hamada et al., 2008; Venken et al., 2011). Because dTRPA1 typically activates neurons only when animals are exposed to temperatures at or above its gating temperature $\left(29^{\circ} \mathrm{C}\right)$, its usage provides perfect genotype-matched controls and bypasses some potential problems that might arise due to chronic activation of neurons during development. We found that activating neurons labeled by the TH-GAL4 driver (Friggi-Grelin et al., 2003) caused females to consistently favor the sucrose substrate over the plain one for egg laying (Fig. $4 A, B$; $d T R P A 1 /+, 32 v s T H>d T R P A 1,32$; $t$ test: $t_{(63)}$ $=29.6, p<0.0001)$, suggesting that these TH-activated females considered the sucrose substrate the superior option of the two. Importantly, females that carried only one of the two transgenes or were assayed at room temperature (where dTRPA1 does not activate neurons) still robustly preferred the plain substrate (Fig. $4 A, B)$, suggesting the preference reversal we observed was indeed due to activation of $\mathrm{TH}$ neurons.

\section{Activating $\mathrm{TH}$ neurons specifically enhanced the value of egg- laying substrates that contained sucrose}

We next assessed whether activating the $\mathrm{TH}$ neurons acts specifically to promote the value of substrates that contain sucrose or whether it reverses females' egg-laying preferences in general. To distinguish between these two possibilities, we examined how females with activated $T H$ neurons might choose in a soft versus firm decision. We noticed that females tended to avoid laying eggs on a firm surface- - hence the lack of egg laying on the plastic divider in our chambers. Indeed, we found that control females consistently preferred to lay eggs on the softer option when given a choice between a soft (1.0\% agarose) and a firm (1.8\% agarose) option (Fig. 5A). TH-activated animals showed a preference for the softer option also $(T H /+, 32, v s T H>d T R P A 1,32$; $t$ test: $t_{(62)}=1.47, p=0.15$; Fig. $5 A$ ), suggesting that activating the $\mathrm{TH}$ neurons does not reverse females' egg-laying preferences in general.

We then tested how TH-activated females responded to the firm option if sucrose had been added to it. If activating the $T H$ neurons can indeed increase the value of substrates that contain sucrose, we would expect $\mathrm{TH}$-activated females to start accepting the firm-and-sweet option (firm sweet ), even if they would normally reject the firm option. Indeed, TH-activated females showed a significant acceptance of the firm sweet $_{\text {option when }}$

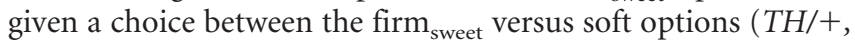
32 vs $T H>d T R P A 1,32$; $t$ test: $t_{(17)}=6.35, p<0.0001$; Fig. $\left.5 B\right)$. This result is consistent with the notion that activating the $\mathrm{TH}$ neurons increases the value of egg-laying substrates that contain sucrose.

What are the consequences of inhibiting the TH neurons on the sucrose versus plain decision? If activating the $T H$ neurons increases the value of the sucrose substrate, inhibiting them should result in females favoring the plain option still. We inhib- 


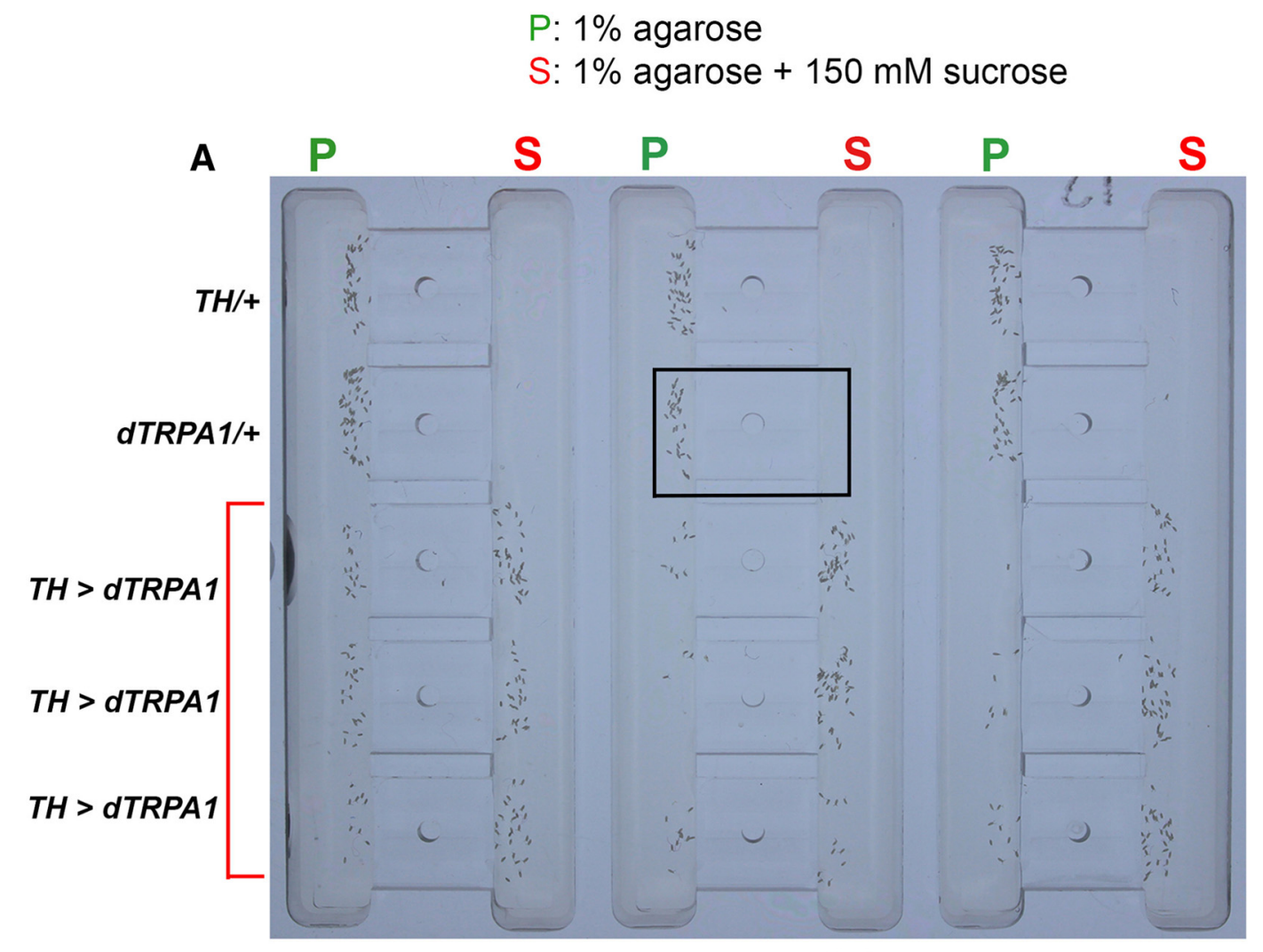

\author{
$\mathrm{P}: 1 \%$ agarose \\ S: $1 \%$ agarose $+150 \mathrm{mM}$ sucrose
}

B

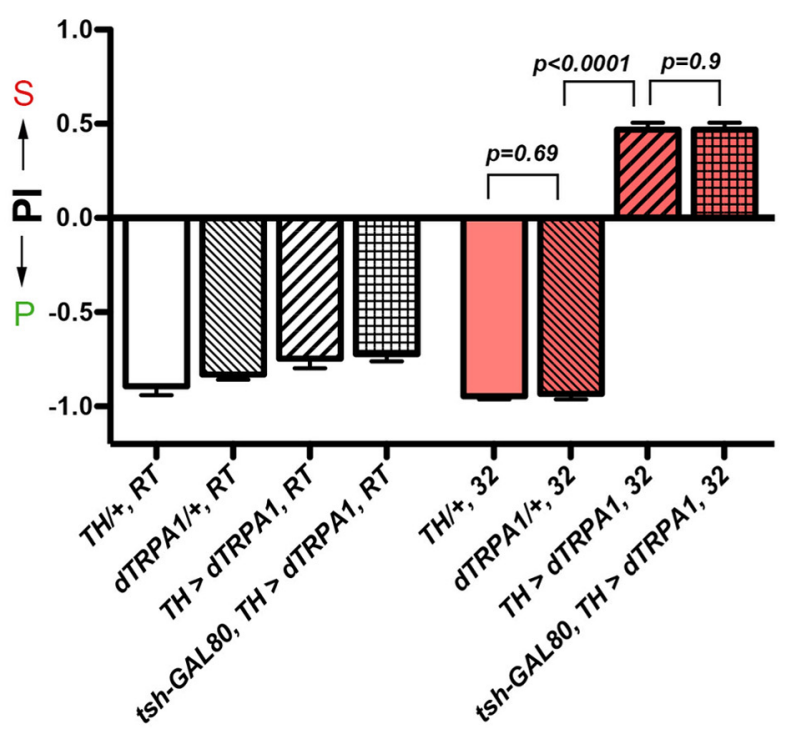

Figure 4. Activating the TH-GAL4-expressing neurons triggered a preference for laying eggs on the sucrose substrate in our sucrose $(S)$ versus plain (P) chambers. $A$, Picture showing that when assayed at $32^{\circ} \mathrm{C}$, control animals (top two rows) preferred to lay eggs on the plain substrates whereas animals with dTRPA1 expressed in their TH neurons (bottom three rows) preferred to lay eggs on the sucrose substrate. The boxed area in the middle denotes the area of a single egg-laying chamber. $B$, Quantification of the effect of activating $T H$ neurons on egg-laying preferences. Positive Preference Index (PI) indicates a preference for the sucrose substrate. The following abbreviations are used: (1) TH/+: TH-GAL4/+, (2) dTRPA1/+: UAS-dTRPA1/+, (3) TH > dTRPA1: UASdTRPA1/+; TH-GAL4/+, and (4) tsh-GAL80, TH > dTRPA1: UAS-dTRPA1/tsh-GAL80; TH-GAL4/+. RT, assayed at room temperature; 32, assayed at 32 ${ }^{\circ} \mathrm{C} . \mathrm{N}=15,42,35,31,33,23,43,53$ for columns, left to right. Also, in $\boldsymbol{B}$ and the rest of the figures that contain multiple data entries, we show the statistics and the $p$ values of only the most relevant comparisons.

ited the $T H$ neurons by expressing in them the widely used temperature-sensitive shibire ${ }^{T S}$, a dynamin mutant that can inhibit synaptic transmission at the restricted temperatures (Kitamoto, 2001; Venken et al., 2011). Curiously, inhibiting $T H$ neurons with shibire ${ }^{T S}$ lowered females' egg-laying rate significantly - most laid only one to two eggs overnight, and did so even when we introduced a tsh-GAL80 transgene (Clyne and Miesenbock, 2008) to suppress the TH-GAL4 activity in the ventral nerve cord, the insect equivalent of the spinal cord. This result suggests that some of the TH-GAL4-expressing neurons in the brain might be essential for controlling egg-laying rate. Regardless, the very few $T H$-inhibited animals that did lay more eggs $(\sim 10-30$ 
A

firm vs. soft firm: $1.8 \%$ agarose soft: $1.0 \%$ agarose

firmsweet VS. soft

firmsweet: $1.8 \%$ agarose + sucrose soft: $1.0 \%$ agarose

sucrose vs. plain sucrose: $1.0 \%$ agarose + sucrose plain: $1.0 \%$ agarose

B

C
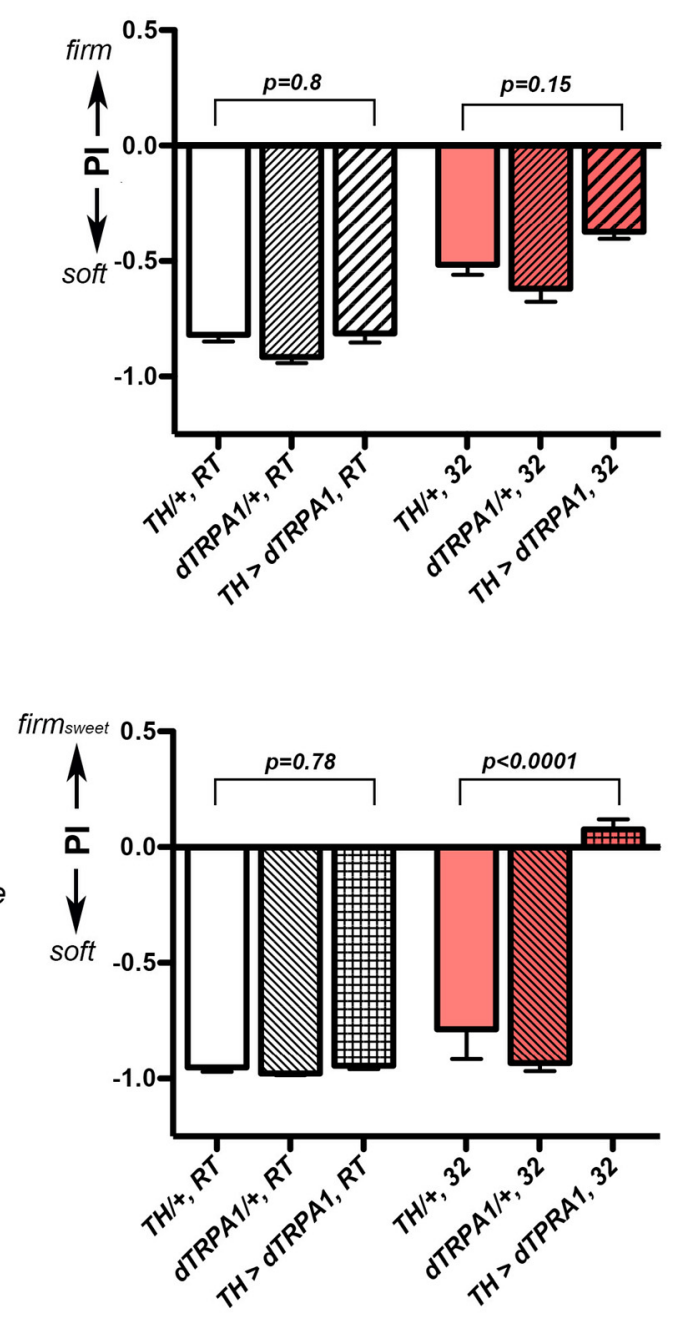

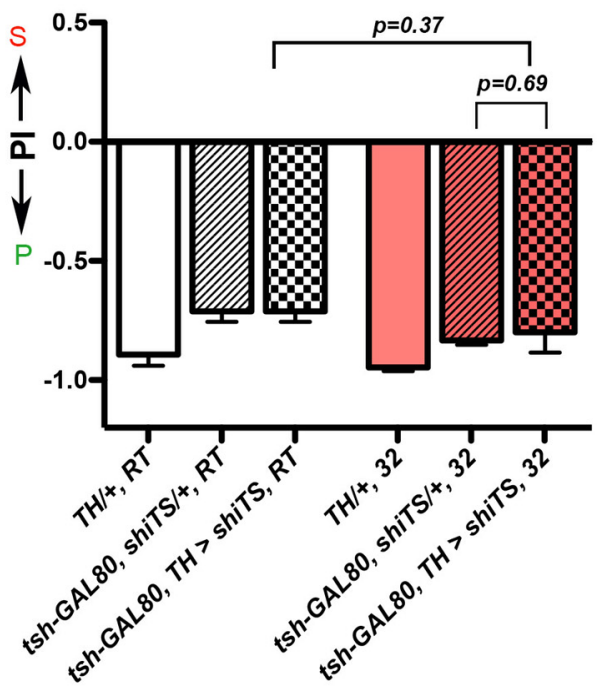

Figure 5. Activation of $T H$-GAL4-expressing neurons did not alter females' egg-laying preferences in general. $\boldsymbol{A}$, Females with their $T H$ neurons artificially activated continued to prefer laying eggs on the soft substrate in a firm versus soft assay. Positive Preference Index (PI) indicates a preference for the firm substrate. $N=31,30,22,36,34,41$ for columns, left to right. $\boldsymbol{B}$, Activating the TH-GAL4 neurons increased egg-laying acceptance of a firm substrate that contained $150 \mathrm{~mm}$ sucrose. Positive PI indicates a

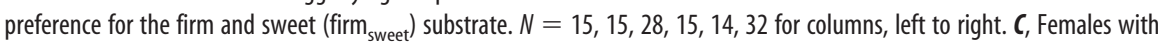
inhibited $T H$ neurons still preferred the plain substrate in the sucrose versus plain assay. Positive PI indicates a preference for the sucrose substrate. The following abbreviations are used: (1) tsh-GAL80, shiTS/+: tsh-GAL80/+; UAS-shibire TS $_{+}+$and (2) tshGAL80, TH > shiTS: tsh-GAL80/+;TH-GAL4/UAS-shibire ${ }^{T S} . N=15,59,59,33,60,19$ for columns, left to right. S, sucrose; $P$, plain.

eggs overnight) still rejected the sucrose substrate robustly ( $t$ sh-GAL80, shiTS/+, 32 vs tsh-GAL80, TH > shiTS, 32; $t$ test: $t_{(19)}=0.4, p=0.69$; Fig. $\left.5 C\right)$, in keeping with the notion that $\mathrm{TH}$ neurons promote the value of sucrose-containing substrates for egg laying.

TH-activated animals actively suppressed laying eggs on the plain substrate in our sucrose versus plain decision chambers

We next investigated the potential behavioral strategy $\mathrm{TH}$-activated animals used to preferentially reject the plain substrate when making the sucrose versus plain decisions. First, we found that $\mathrm{TH}$-activated animals readily laid eggs on the plain substrate when it was the sole option (ANOVA: $F_{(2,12)}=0.70, p=0.51$; Fig. $6 A)$, suggesting that they considered the plain option acceptable for egg laying. Second, in the sucrose versus plain assay, $\mathrm{TH}$-activated females regularly visited the plain substrate during the one-back period (Fig. $6 B$ ) before most ELEs ( $80 \%$ of the 235 ELEs), and did so when they should be physically ready to deposit another egg $\left(t\right.$ test: $t_{(345)}=11.31, p<0.0001$; Fig. 6D). Moreover, the duration of their last plain visit should be sufficiently long, in principle, to have allowed the egglaying motor program to be activated during this visit (Fig. $6 C, E$; $t$ test: $t_{(384)}=0.13$, $p=0.89$ ). Thus, collectively, these results suggest that $T H$-activated animals' rejection of the plain substrate was the result of actively suppressing laying eggs on it, after they had learned the presence of the sucrose substrate in the chambers.

Our results suggest a model that activating $\mathrm{TH}$ neurons increases the value of substrates that contain sucrose, triggering TH-activated females to consider the sucrose substrate the superior option. Consequently, while TH-activated females still accepted the plain substrate when it was the sole option, they tended to reject it when the sucrose option was available. Moreover, they executed their decision to reject the plain option by suppressing their egg-laying motor program when visiting it, mirroring the behavioral strategy wild-type females used to reject the sucrose substrate for egg laying.

\section{Activation of DA neurons promoted a} preference for laying eggs on the sucrose substrate in our sucrose versus plain decision chambers

We next wanted to identify the relevant TH-GAL4-expressing neurons whose activation was responsible for increasing the 
A
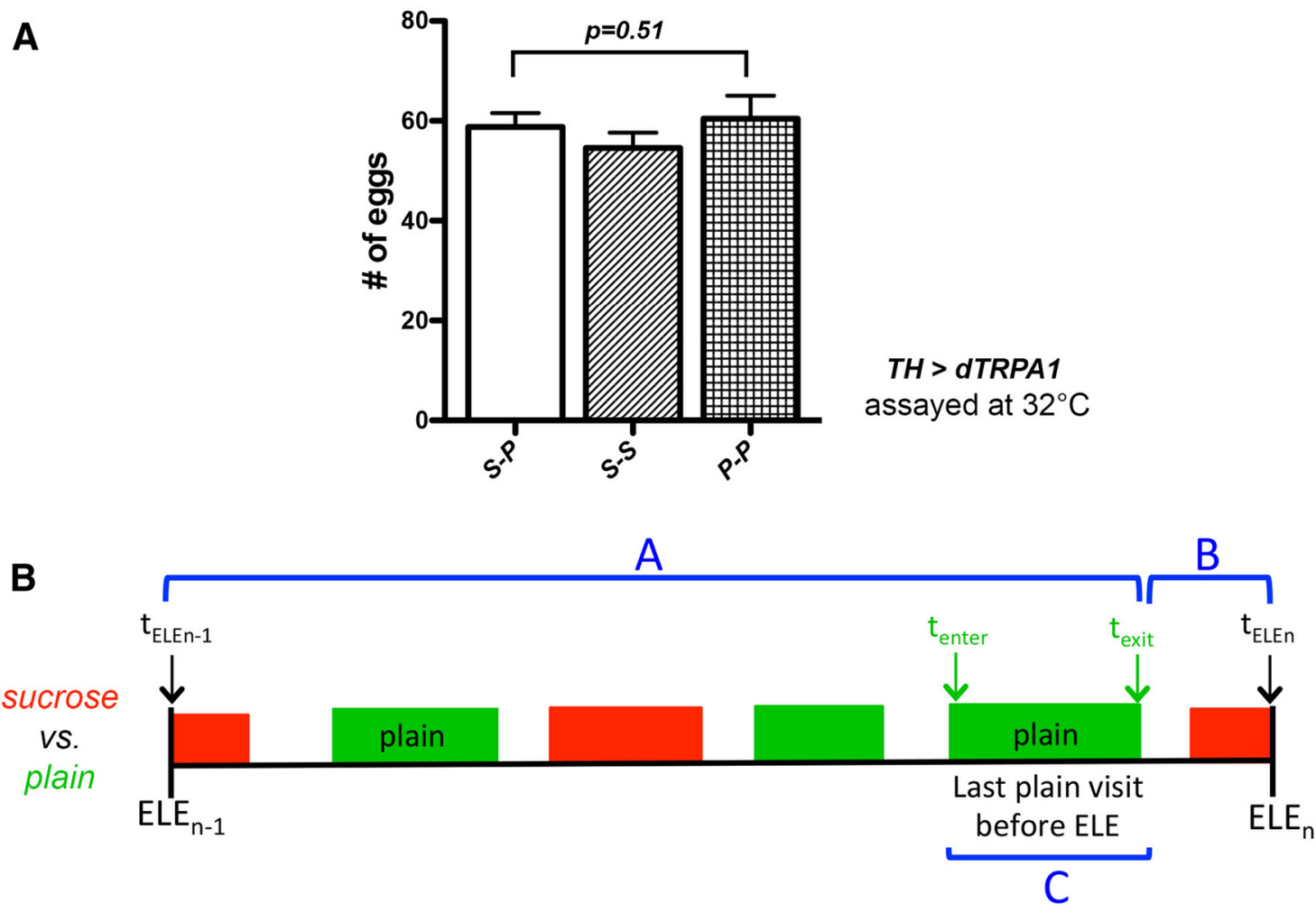

C

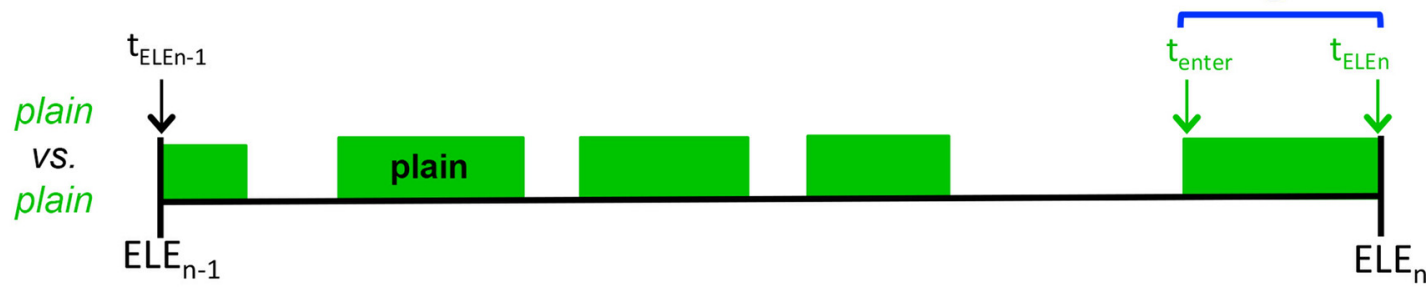

D

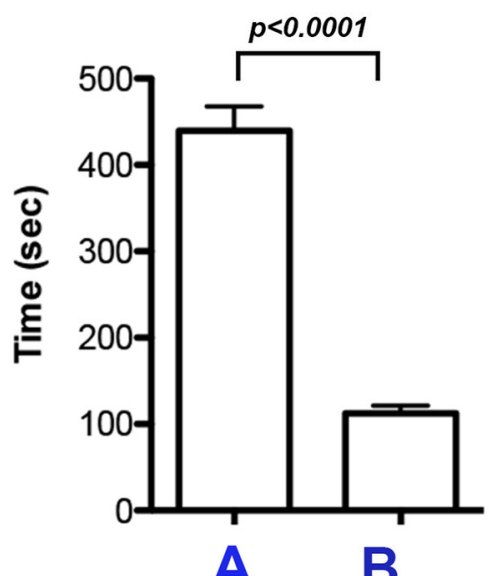

$\mathbf{E}$

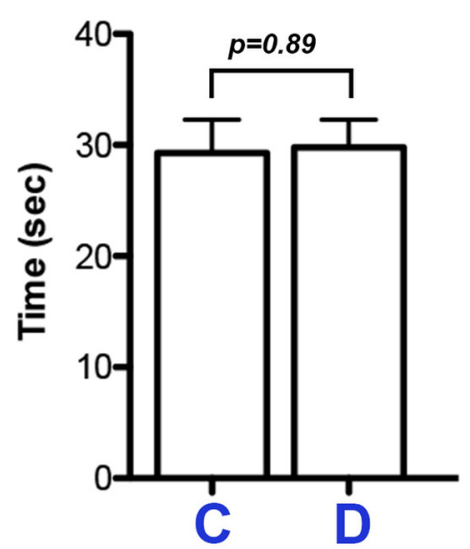

Figure 6. TH-activated females continued to visit the plain substrate in our sucrose versus plain chambers, and these visits should have allowed egg laying. $A$, $T H$-activated females laid similar numbers of eggs in the sucrose versus plain (S-P), sucrose versus sucrose (S-S), and plain versus plain (P-P) assays. $N=10$ for each. $\boldsymbol{B}$, Schematic diagram depicting the one-back period before an ELE on sucrose when $T H$-activated females were assayed in the sucrose versus plain chambers. $t_{\text {enter }}$ and $t_{\text {exit }}$ time of entrance and exit of the plain substrate during the last plain visit, respectively; duration $A$, elapsed time between $t_{E L E n-1}$ and $t_{\text {exit }}$; duration $B$, elapsed time between $t_{\text {exit }}$ and $t_{\mathrm{ELEn}}$; duration C, duration of the last plain visit. $C$, Schematic diagram depicting the one-back period before an ELE when $T H$-activated females were assayed in the plain versus plain chambers; duration $\mathrm{D}$, elapsed time between $\mathrm{t}_{\text {enter }}$ and $\mathrm{t}_{\mathrm{ELEn}}$. It reflects the time of contact with the plain substrate females need to activate the egg-laying motor program on this substrate. $D$, The time when a $T H$-activated female exited the plain substrate during the last plain visit was closer to the time of $E_{E} E_{n}$ than $E_{E E_{n-1}}$. Columns $A$ and $B$; see also diagram in panel B.N $=292$ for column A and 179 for column B. $\boldsymbol{E}$, A comparison of the amount of time a $\mathrm{TH}$-activated female spent on the last plain visit (column C) to the time of contact it needs to activate the egg-laying motor program on the plain substrate (column D). $N=179$ for column C, 267 for column D. 
value of the sucrose-containing substrates for egg laying. Because TH-GAL4 mainly labels DA neurons (Friggi-Grelin et al., 2003; Mao and Davis, 2009), we first asked whether DA neurons are the relevant players. We reduced expression of four known DA receptors individually in TH-activated animals (Han et al., 1996; Hearn et al., 2002; Thibault et al., 2004; Kim et al., 2007; Keleman et al., 2012; Q. Liu et al., 2012; Marella et al., 2012) and found that while reducing the dopaminergic receptor $D o p R$ did not reduce their sucrose preference, reducing the three other receptors, $D R 2, D 2 R$, or DopEcR, $\operatorname{did}\left(T H^{*}, 32 v s T H^{*}\right.$ in DopR, 32; $t$ test: $t_{(116)}=0.31, p=0.76 ; T H^{*}$, 32 vs $T H^{*}$ in DR2, 32; $t$ test: $t_{(94)}=5.78, p<0.0001 ; H^{*}, 32 v s H^{*}$ in DopEcR, 32, $t$ test; $t_{(104)}=12.1, p<0.0001$; Figure $7 A$ ). This result suggests $D A$ neurons are the relevant players because activating $T H$ neurons failed to promote proper sucrose preference when functions of $D A$ receptors were compromised.

Past studies have suggested that there are at least eight distinct clusters of DA neurons in each adult brain hemisphere and one unpaired cluster in the subesophageal ganglion (SOG) of the brain (Mao and Davis, 2009; Kong et al., 2010). Different clusters of DA neurons have been shown to regulate different behaviors that range from olfactory learning to sleep-wake control (T. Liu et al., 2008; Kong et al., 2010; Burke et al., 2012; Inagaki et al., 2012; Keleman et al., 2012; C. Liu et al., 2012; Q. Liu et al., 2012; Marella et al., 2012; Azanchi et al., 2013). In particular, activation of a specific DA neuron in the SOG, the TH-VUM neuron, has been shown to enhance hungry flies' proboscis extension reflex when they detect sucrose (Marella et al., 2012). The ability of the TH-VUM neuron to enhance the sucrose-induced feeding reflex prompted us to ask whether it also promotes egg-laying acceptance of sucrose. To test this, we obtained the $D d c(H L-5)-G A L 4$, which has been suggested to label DA neurons in the SOG (Kong et al., 2010) and we confirmed that it indeed labeled all the DA neurons (i.e., neurons that express the $\mathrm{TH}$ protein) in the SOG that we could detect, including the TH-VUM neuron (Figs. $7 B, D-F, 8 F$ ). Activating the Ddc(HL5)GAL4 neurons did not trigger any preference for laying eggs on the sucrose substrate (HL5 > dTRPA1, RT vs HL5 > dTRPA1, 32; $t$ test: $t_{(80)}=2.37, p=0.02$; Fig. $7 C$ ), however, arguing against the possibility that TH-VUM is the sole player in enhancing the value of the sucrose-containing substrates for egg laying.

\section{Activation of DA neurons labeled by the $C^{\prime}-G A L 4$ promoted a preference for laying eggs on sucrose in the sucrose versus plain decision chambers}

We next tested a collection of newly generated GAL4 drivers, each of which labels a subset of DA neurons (Q. Liu et al., 2012). We found that activating the neurons labeled by one of the GAL4 s, the $C^{\prime}-G A L 4$, produced a substantial increase in sucrose acceptance for egg laying (tsh-GAL80, dTRPA1/+, $32 v s C^{\prime}>t s h$ GAL80, dTRPA1, 32; $t$ test: $t_{(35)}=18, p<0.0001$; Fig. $\left.8 A\right)$. We then used the $C^{\prime}$-GAL4 to express a green fluorescent marker $(m C D 8-G F P)$ and found that while it labeled neurons relatively sparsely in the adult brain (Fig. $8 C$ ), it consistently marked the DA neurons that belong to the SOG, PAL, and PPL2 clusters (Fig. $8 C-F)$, suggesting that DA neurons in the PAL and the PPL2 are candidates for promoting sucrose preference during egg laying. However, Ddc(HL5)-GAL4 has been reported to label DA neurons in the PAL and the PPL2 clusters also (Kong et al., 2010), but it did not promote any sucrose preference in our assays (Fig. 7C). To resolve this contradiction, we examined the labeling patterns of TH-GAL4, C'-GAL4 and Ddc(HL5)-GAL4 side by side. We found that $D d c$ (HL5)-GAL4 consistently labeled fewer DA neurons in the PPL2 and PAL clusters than $C^{\prime}-G A L 4$ or TH-GAL4 did (Fig. $8 F$ ), in support of the notion that PPL2 and PAL2 are reasonable candidates for promoting the value of sucrose substrates for egg laying. However, we could not rule out that other $C^{\prime}$-GAL4-expressing neurons might also contribute.

We also examined the impact of inhibiting $C^{\prime}$-GAL4-expressing neurons on egg-laying decisions. In contrast to females with inhibited TH-GAL4 neurons, females with inhibited $C^{\prime}$-GAL4 neurons did not reduce their egg-laying rate significantly $\left(C^{\prime}>t s h\right.$-GAL80, shiTS, $R T$ vs $C^{\prime}>$ tsh-GAL80, shiTS, $32 ; 47 \pm 2.6$ vs $42 \pm 2.5, t$ test: $\left.t_{(54)}=1.4, p=0.17\right)$. Interestingly, females with shibire ${ }^{T S}$ expressed in their $C^{\prime}$-GAL4 neurons showed a stronger sucrose rejection phenotype when assayed at $32^{\circ} \mathrm{C}$ than when assayed at room temperature $\left(C^{\prime}>\right.$ tsh-GAL80, shiTS, $R T$ vs $C^{\prime}>$ tshGAL80, shiTS, 32; $t$ test: $t_{(43)}=4.79, p<0.0001$; Fig. $\left.8 B\right)$. In contrast, the two controls showed comparable rejection at the two temperatures $\left(C^{\prime} /+, R T\right.$ vs $C^{\prime} /+, 32$; $t$ test: $t_{(55)}=1.62, p=$ 0.11; tsh-GAL80, shiTS/+, RT vs tsh-GAL80, shiTS/+, 32; $t$ test: $t_{(33)}=0.27, p=0.78$; Figure $8 B$ ). These results suggest DA neurons labeled by the $C^{\prime}$-GAL4 can modulate the value of the sucrose substrates as options for egg laying.

\section{The MB did not play an essential role in mediating the sucrose versus plain egg-laying decision}

Finally, we were interested in identifying the specific brain center that reads and ranks the values of egg-laying substrates when females are deciding between the sucrose versus plain options. Several lines of evidence suggest the MB is a reasonable candidate. First, it is one of the targets of the PPL2 DA neurons (Mao and Davis, 2009). Second, it is a well documented learning and memory center and has also been shown to mediate several decisionmaking tasks (Zhang et al., 2007; Joseph et al., 2009; Azanchi et al., 2013; Lin et al., 2014). Third, its ability to decode the DA signals has been reported to be important for mediating ethanol versus no ethanol decisions (Azanchi et al., 2013).

To determine whether the $\mathrm{MB}$ indeed plays a role in mediating the sucrose versus plain decision, we first physically ablated it by using a commonly used HU protocol (de Belle and Heisenberg, 1994). HU treatment ablated the MB in almost all the samples we looked at (34/35; Fig. 9A,B), but the ablated and untreated animals showed the same egg-laying preferences, with or without their DA neurons activated by dTRPA1 (WT vs HU, WT; $t$ test: $t_{(55)}=0.29, p=0.77$; Fig. $9 \mathrm{C}$; TH $>d T R P A 1,32$ vs $\mathrm{HU}, \mathrm{TH}>$ $d T R P A 1,32$; $t$ test: $t_{(133)}=1.18, p=0.24$; Figure $\left.9 D\right)$. To further confirm this finding, we also inactivated the synaptic output of the MB neurons. This was accomplished by using the LexA version of a commonly used $\mathrm{MB}$ driver (247-LexA) to express in the $\mathrm{MB}$ neurons the temperature-sensitive synaptic inhibitor shibire $^{T S}$ (LexAop-shiTS; Lin et al., 2014). LexA/LexAop is a commonly used binary gene expression system in flies (Lai and Lee, 2006) and can work independently from the GAL4/UAS system, allowing us to inhibit the MB neurons (with 247-LexA/LexAop$s h i^{T S}$ ) while activating the DA neurons (with TH-GAL4/UASdTRPA1) in the same animal. Again, MB-inhibited and control animals showed the same egg-laying preferences, regardless of whether their DA neurons were thermogenetically activated or $\operatorname{not}\left(247>\operatorname{shiTS}, R T\right.$ vs $247>\operatorname{shiTS}, 32 ; t$ test: $t_{(80)}=0.58, p=$ 0.56; Fig. $9 E$; $T H^{*}, 32 v s T H^{*}, 247>$ shiTS, 32 ; $t$ test: $t_{(31)}=$ $0.12, p=0.93$; Figure $9 F)$. These results suggest that $\mathrm{MB}$ does not play an essential role in mediating the sucrose versus plain egg-laying decision in our chambers. However, we cannot rule out that it acts in parallel with other brain loci to regulate this decision. 
A
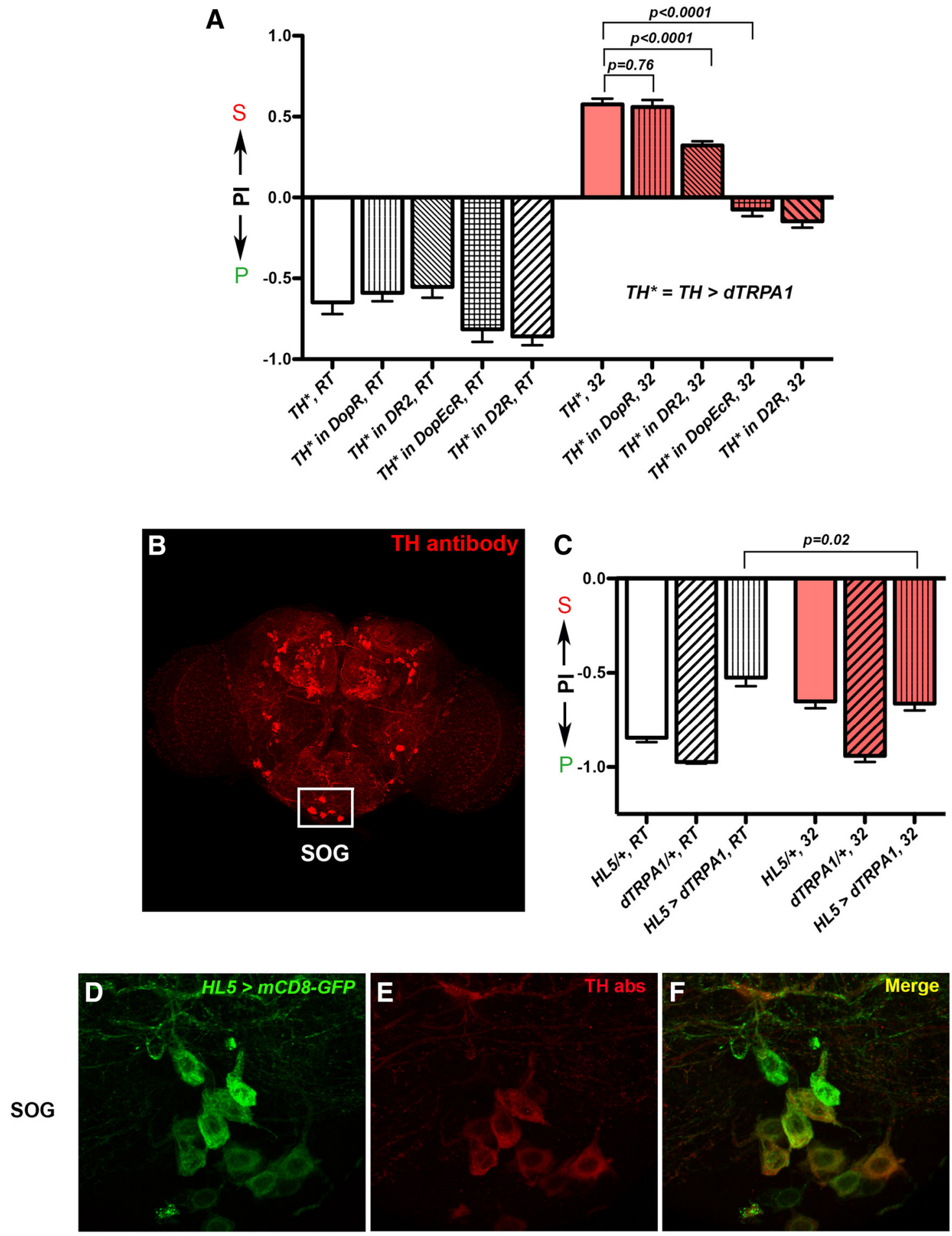

Figure 7. DA neurons are candidates for promoting a preference for laying eggs on the sucrose substrate. $A$, Reducing the dopamine receptors $D R 2, D 2 R$, or $D o p E c R$ reduced the ability of $T H$ neurons to promote sucrose preference, but reducing the receptor DopR had no effect. The following genotype abbreviations are used: (1) TH* in DopR: UAS-dTRPA1/+;DopR1, TH-GAL4/DopR1, (2) TH* in DR2:UAS-dTRPA1/+;DR2, TH-GAL4/DR2, (3) TH* in DopEcR:UAS-dTRPA1/+;DopECR, TH-GAL4/DopECR, and (4) TH*in D2R:D2R;UAS-dTRPA1/+;TH-GAL4/+.N = 23,65, 34, 29, 70, 46, 73, $79,61,70$ for columns, left to right. $B$, Antibody staining of TH in an adult female brain. Neurons in the boxed area are $T H$ neurons that reside in the SOG area. $C$, Activating Ddc(HL5)-GAL4-expressing neurons did not trigger a preference for laying eggs on the sucrose substrate. The following abbreviations are used: (1) HL5/+:Ddc(HL5)-GAL4/+ and (2) HL5 > dTRPA1: Ddc(HL5)-GAL4/+; UAS-dTRPA/+.N = 34, 19,43, 43, 18, 43 for columns, left to right. D-F, Ddc(HL5)-GAL4 labeled all the TH antibody-positive neurons in the SOG. D, Ddc(HL5)-GAL4 neurons labeled by mCD8-GFP. $\boldsymbol{E}$, TH antibody staining of the same neurons as in $\boldsymbol{D}$. $\boldsymbol{F}$, Merged image. See also Figure $8 F$. PI, Preference Index. S, sucrose; $P$, plain.

\section{Discussion}

Using new tools we developed that can assay egg-laying preferences and positional trajectories of females at single-animal resolution and in high throughput, we investigated the neural mechanism that underlies Drosophila females' rejection of the sucrose substrate for egg-laying in a simple sucrose versus plain decision-making task. In keeping with our earlier finding (Yang et al., 2008), we found that females readily deposited their eggs on 
A

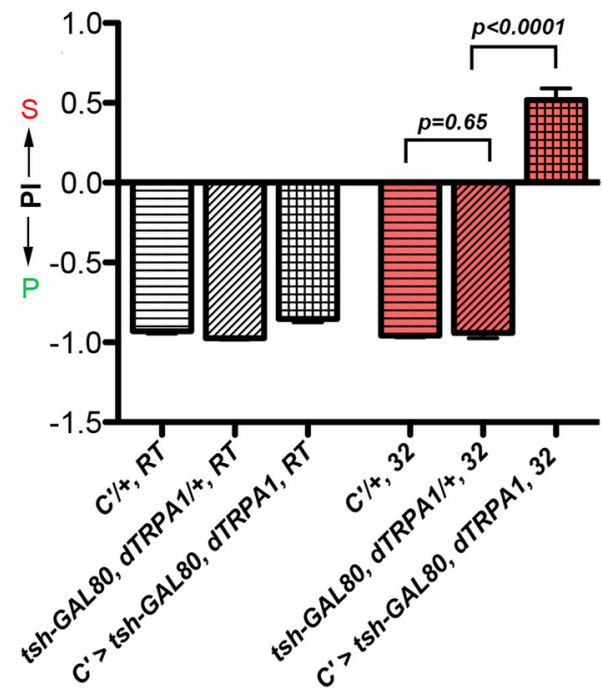

B

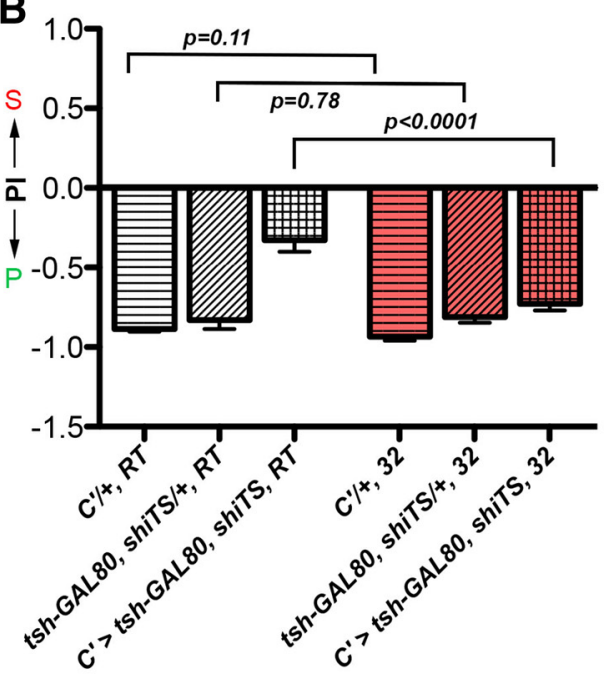

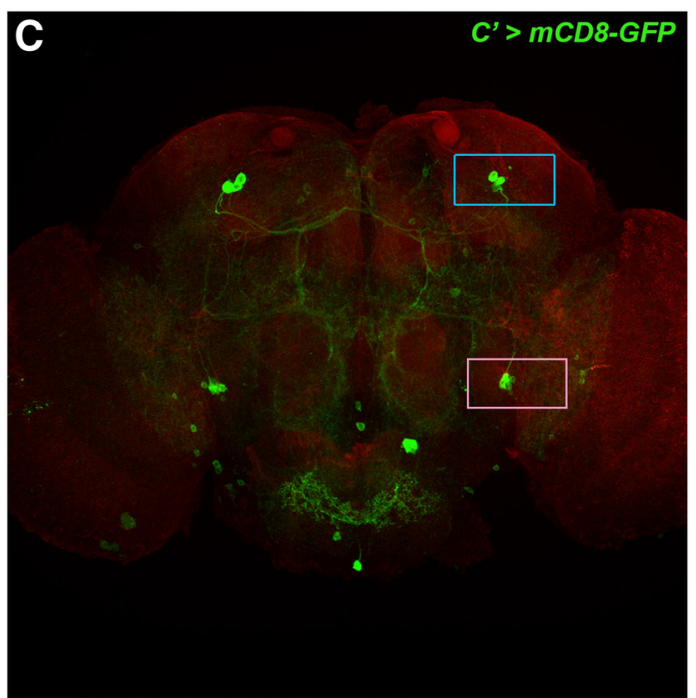
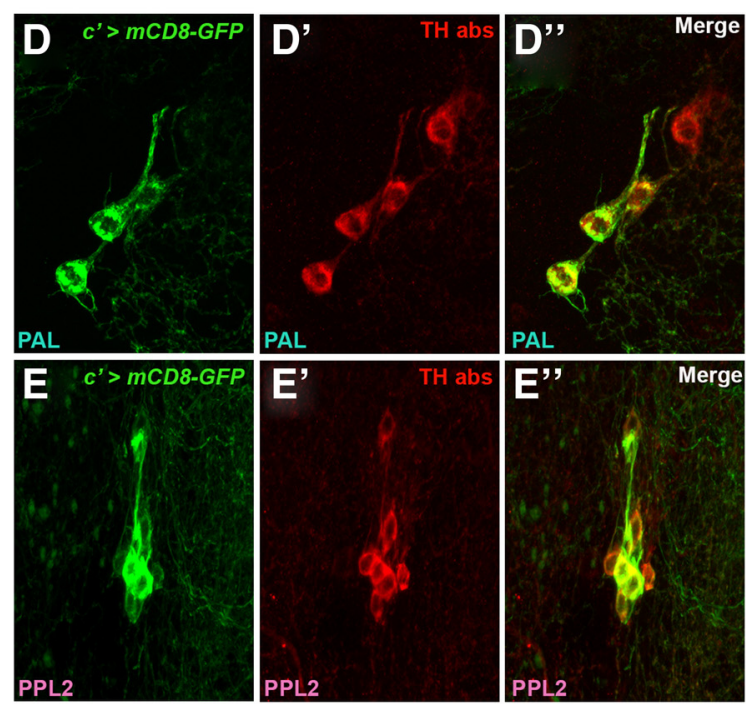

$\mathbf{F}$

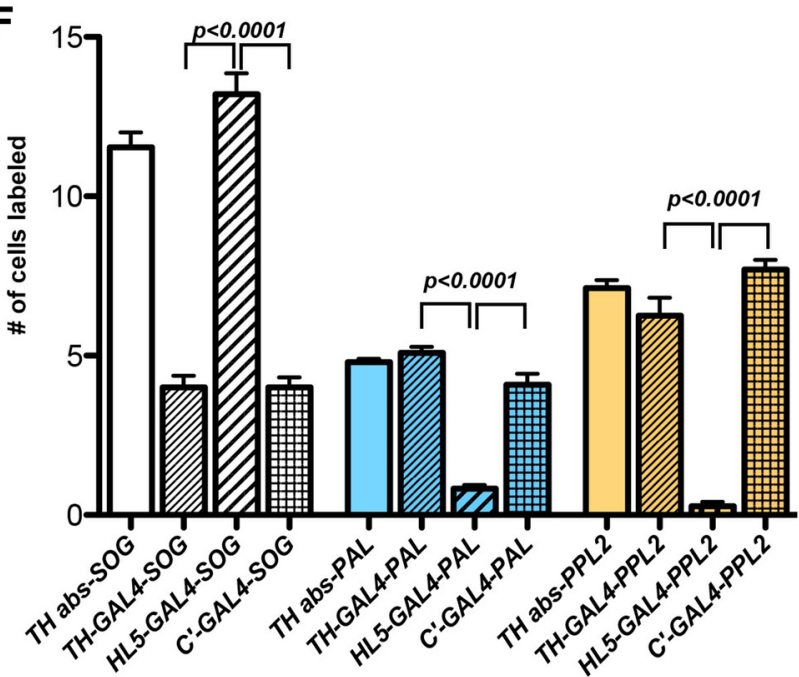

Figure 8. DA neurons labeled by the $C^{\prime}-G A L 4$ are candidates for promoting a preference for laying eggs on the sucrose substrate. $A$, Females with their $C^{\prime}-G A L 4$-expressing neurons activated preferred the sucrose substrate for egg laying. $N=29,17,36,25,17,27$ for columns, left to right. $B$, Females with their $C^{\prime}-G A L 4$-expressing neurons inhibited preferred the plain substrate for egg laying. $N=29,32,20,28$, 31,25 for columns, left to right. The following abbreviations are used for $A$ and $B$ : (1) $C^{\prime} /+: C^{\prime}-G A L 4 /+,(2) C^{\prime}>$ tsh-GAL80, dTRPA1:tsh-GAL80, UAS-dTRPA1/+, $C^{\prime}-$ GAL4/+, and (3) $C^{\prime}>$ tsh-GAL80, shiTS: tsh-GAL80/+; $C^{\prime}-$ GAL4/UAS-shibire ${ }^{T S}$. C, $C^{\prime}$-GAL4 labeled neurons (in green) sparsely in the adult brain (counterstained with HRP). Blue rectangle, PAL cluster; pink rectangle, PPL2 cluster. D-D $D^{\prime \prime}, C^{\prime}-G A L 4$ neurons in the PAL cluster coexpressed TH. D, $C^{\prime}$-GAL4 neurons labeled by $m$ CD8-GFP. $\boldsymbol{D}^{\prime}$, The same neurons stained with anti-TH antibody. $\mathbf{D}^{\prime}$, Merged image. Note that neurons in these images are different from those shown in $\boldsymbol{C} . \boldsymbol{E}-\boldsymbol{E}^{\prime}, \boldsymbol{C}^{\prime}$-GAL4 neurons in the PPL2 cluster coexpressed TH.E, $\boldsymbol{C}^{\prime}$-GAL4 neurons labeled by $m$ CD8-GFP. $\boldsymbol{E}^{\prime}$, The same neurons stained with anti-TH antibody. $\boldsymbol{E}^{\prime}$, Merged image. Note that neurons in these images are different from those shown in $C$. $F$, Comparison of the numbers of DA neurons in the SOG, PPL2, and PAL clusters that are labeled by TH-GAL4, $C^{\prime}-$ GAL4 and Ddc(HL5)-GAL4, respectively. For each GAL4, six brains were examined. GAL4-expressing neurons were scored by $m$ CD8-GFP expression. DA neurons were scored by TH antibody staining. S, sucrose; $P$, plain; $P$, Preference Index. 
the sucrose substrate when it was the sole option, but rejected it when a plain substrate was available. This result suggests that, in our assay, adding sucrose to an acceptable substrate (e.g., 1\% agarose) decreases the value of this substrate for egg laying. Behavioral analysis allowed us to understand the strategy females used to choose their egg-laying substrate. We found that females continued to visit the lesser valued sucrose substrate before most egg-laying events in the sucrose versus plain assay. In particular, analysis of the timing and duration of these visits suggests that females should, in principle, have been competent to lay eggs on the sucrose substrate. Thus, females must have actively suppressed the activation of their egg-laying motor program during these sucrose visits, but only after learning that a superior plain option was also available. It is unclear when such learning occurred. Females may learn and remember the quality of their options during their initial exploration of the decision chamber and use the "knowledge" to guide all subsequent egg-laying decisions. On the other end of the spectrum, they may learn on an egg-by-egg basis: they relearn the quality of available options during the early phase of each one-back period and use the knowledge to guide only the immediate upcoming egg-laying decision.

How does the female brain transform the sensation of the sucrose substrate into a value that can be ranked? Because the same sucrose substrate can be either inferior or superior to a plain substrate depending on the activation state of the DA neurons, we hypothesize that detection of sucrose in a substrate may engage two distinct pathways: one that increases the value of this substrate for egg laying and another that reduces it. We speculate that in our sucrose versus plain assay, the "value-reducing pathway" is dominant, causing the sucrose substrate to have a lower value than the plain one (and its subsequent rejection), but activating the DA system triggers the "value-increasing pathway" to be dominant instead, causing the sucrose substrate to have a higher value than the plain substrate. The DA system might accomplish this via either inhibiting the value-reducing pathway or facilitating the value-increasing pathway, or both. The DA neurons we describe in this work have been shown to project to diverse loci in the brain (Mao and Davis, 2009); identifying the critical members within these DA neurons and mapping their targets will be the crucial

E
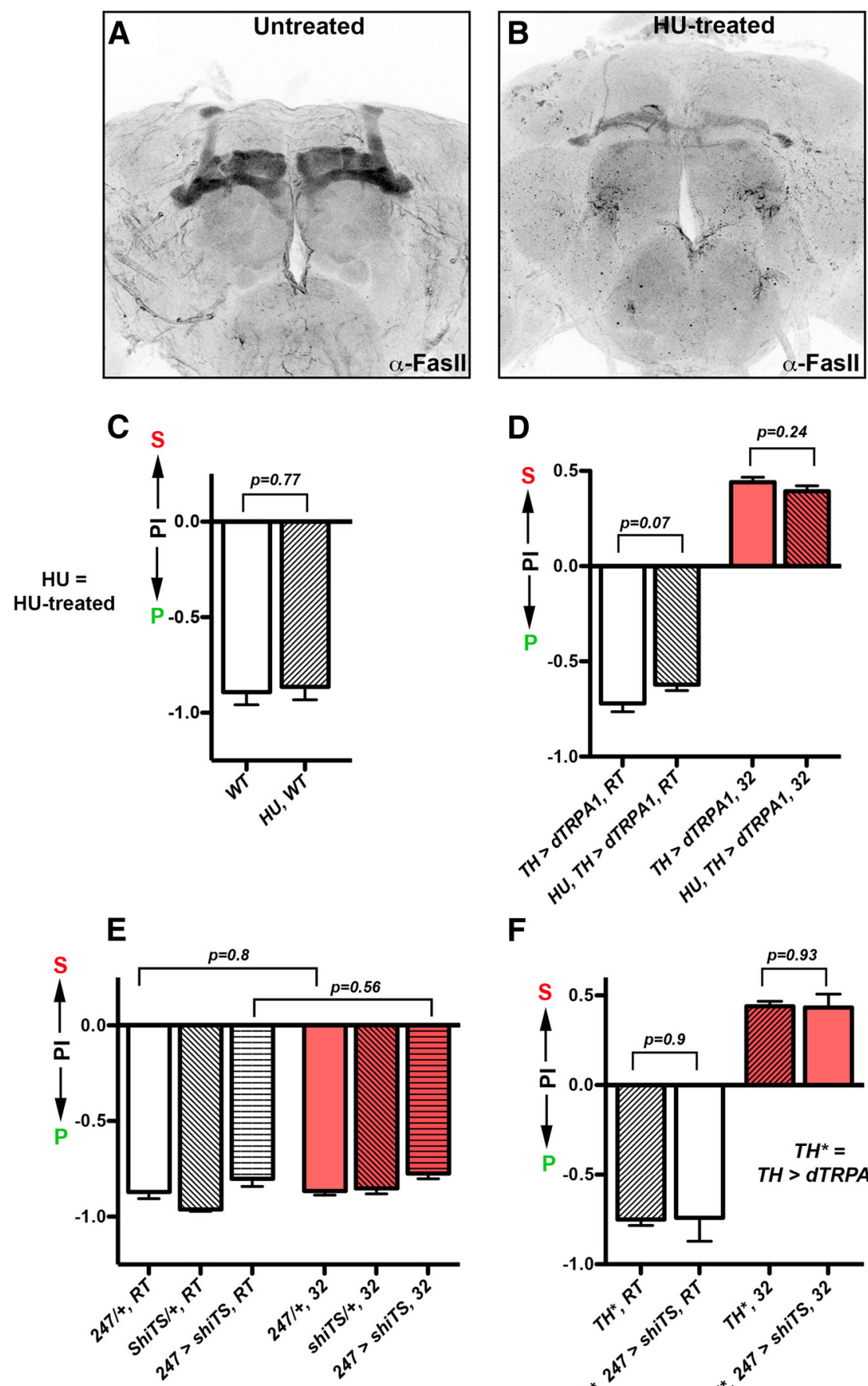

$\mathbf{F}$

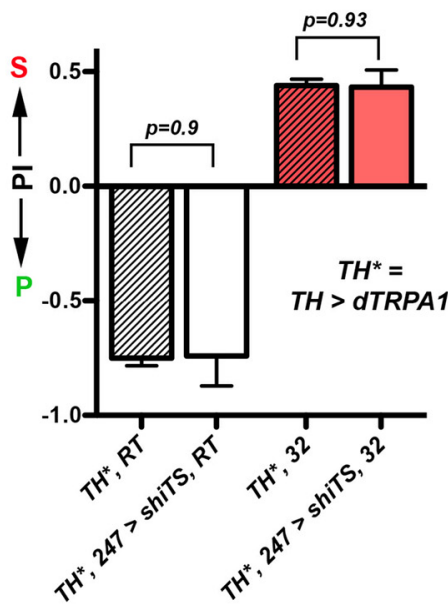

Figure 9. Selective ablation and inhibition of MB neurons did not affect females' appraisal of sucrose substrate for egg laying. $A$, A representative picture showing that $M B$ was intact in the brains of untreated animals (34/34). $\boldsymbol{B}$, A representative picture showing that MB was largely ablated in the brains of HU-treated animals (34/35). C, HU-treated and untreated WT flies showed similar egg-laying preferences for the plain substrate. $N=30$ for each group. $\boldsymbol{D}$, HU-treated and untreated $T H$-activated flies showed similar egg-laying preferences for the sucrose substrate. $N=41,45,68,68$ for columns, left to right. $\boldsymbol{E}$, Inhibiting the output of MB neurons did not affect females' preference for laying eggs on the plain substrate. The following abbreviations are used: (1) 247/+: 247-LexA/+, (2) shiTS/+:LexAop-shibire $/+$, and (3) 247 > shiTS:LexAop-shibire $/+; 247-$ LexA/+. N = 27, 25, 45, 30, 28, 51 for columns, left to right. $\boldsymbol{F}$, Inhibiting the output of MB neurons did not affect $T H$-activated females' preference for laying eggs on the sucrose substrate. The following abbreviations are used: (1) TH*: UAS-dTRPA1/+;TH-GAL4/+ and (2) TH*, $247>$ shiTS: LexAop-shibire ${ }^{\text {TS }} /+$; UAS-dTRPA1/+; TH-GAL4/247-LexA.N = 40, 25, 67, 20 for columns, left to right. S, sucrose; $P$, plain; PI, Preference Index.

next step to test the validity of this hypothesis and to begin delineating the circuit that underlies the value transformation process.

It is worth pointing out that, in our assay, the DA system appears to modify the value of sucrose substrates specifically- 
increasing DA activity did not impact how females chose between options that lacked sucrose and differed only in texture. Such specificity, in combination with earlier findings, suggests a potential division-of-labor scheme of the Drosophila DA system such that different groups of DA neurons mediate different influences of sucrose on behaviors. For example, the VUM-TH neuron enhances the ability of sucrose to trigger proboscis extension (Marella et al., 2012). The PAM-DA cluster enables the ability of sucrose to assign positive valence to neutral odors during appetitive olfaction learning (Burke et al., 2012; C. Liu et al., 2012). The DA cluster(s) we describe here acts to enhance the value of the sucrose substrates for egg laying. It will be interesting to examine whether such division-of-labor scheme is conserved-DA neurons in the vertebrate VTA might also contain distinct subsystems that each processes a distinct behavior-modifying property of rewards.

How does the female brain rank the values of the available egg-laying options? The design of our assay ensures that females cannot simultaneously contact both egg-laying substrates. Thus, their decision to refrain from laying eggs on the inferior but acceptable substrate requires that they memorize the values of their options. The MB is an attractive candidate for storing and ranking the values of egg-laying options. It is a well known learning and memory center in Drosophila and has been shown to receive DA input and mediate several decision-making tasks (Zhang et al., 2007; Joseph et al., 2009; Joseph and Heberlein, 2012; Azanchi et al., 2013; DasGupta et al., 2014). Surprisingly, our results suggest that neither its physical presence nor its synaptic output is essential for mediating the sucrose versus plain decision, though we cannot rule out that it may act in parallel with other brain loci to perform this decision task.

How does the female brain translate the result of its value ranking into behavior output? Our analysis suggests females execute the decision by actively suppressing the egg-laying motor program when exploring the inferior substrate. This suggests that the value ranking brain center must have access to the egg-laying motor program. The identity of the command neurons that control the egg-laying motor program is not known, but it is interesting to note that we have found that inhibiting the TH-GAL4 neurons (but not the $C^{\prime}$-GAL4 neurons) caused females to reduce their egg-laying rate significantly, suggesting that some of the $T H$ neurons are potential positive regulators of the egg-laying motor program. If true, it is possible that the value-ranking brain center may suppress egg laying on the inferior substrates by reducing the activities of these $T H$ neurons.

Finally, why did evolution preserve a mechanism that allows Drosophila females to reject a sucrose substrate over a plain one for egg laying in some contexts? Such rejection seems puzzling at first because sugar is a food source for flies, but it is important to note that the goal of egg-laying females is to choose a site that maximizes the chances of their eggs to develop into reproductively active adults. Food availability, while important, is probably not the sole determinant of egg and larva survival in nature. For example, food-containing sites may attract predators. Moreover, females often remain immobile for a while on the substrate where they just laid an egg and thus are susceptible to predation during this period. Thus, in contexts where food is within reach for the larvae, it may be advantageous for females to forgo the sugary option and choose the sugar-free one nearby instead. What constitutes a context where "a sugary option is within reach of a sugar-free one?" Our previous report suggests that the physical distance between the two substrates matters (Yang et al., 2008), however, results from a recent study (Schwartz et al., 2012) hint at the possibility that the frequency by which females encounter the sucrose option may be more important-if the two substrates were placed close together but in a larger enclosure, females appeared to prefer the sucrose substrate. It should be interesting to test whether an increased frequency of sucrose encounters suppresses the activity of the DA neurons we described, devaluing the sucrose substrate for egg-laying in our sucrose versus plain chambers.

\section{References}

Azanchi R, Kaun KR, Heberlein U (2013) Competing dopamine neurons drive oviposition choice for ethanol in Drosophila. Proc Natl Acad Sci U S A 110:21153-21158. CrossRef Medline

Brand AH, Perrimon N (1993) Targeted gene expression as a means of altering cell fates and generating dominant phenotypes. Development 118 : 401-415. Medline

Branson K, Robie AA, Bender J, Perona P, Dickinson MH (2009) Highthroughput ethomics in large groups of Drosophila. Nat Methods 6:451457. CrossRef Medline

Burke CJ, Huetteroth W, Owald D, Perisse E, Krashes MJ, Das G, Gohl D, Silies M, Certel S, Waddell S (2012) Layered reward signalling through octopamine and dopamine in Drosophila. Nature 492:433-437. CrossRef Medline

Dahanukar A, Lei YT, Kwon JY, Carlson JR (2007) Two Gr genes underlie sugar reception in Drosophila. Neuron 56:503-516. CrossRef Medline

DasGupta S, Ferreira CH, Miesenböck G (2014) FoxP influences the speed and accuracy of a perceptual decision in Drosophila. Science 344:901-904. CrossRef Medline

de Belle JS, Heisenberg M (1994) Associative odor learning in Drosophila abolished by chemical ablation of mushroom bodies. Science 263:692695. CrossRef Medline

Dweck HK, Ebrahim SA, Kromann S, Bown D, Hillbur Y, Sachse S, Hansson BS, Stensmyr MC (2013) Olfactory preference for egg laying on citrus substrates in Drosophila. Curr Biol 23:2472-2480. CrossRef Medline

Friggi-Grelin F, Coulom H, Meller M, Gomez D, Hirsh J, Birman S (2003) Targeted gene expression in Drosophila dopaminergic cells using regulatory sequences from tyrosine hydroxylase. J Neurobiol 54:618-627. CrossRef Medline

Glimcher PW, Camerer CF, Fehr E, Poldrack RA (2009) Neuroeconomics: decision making and the brain, Ed 1. San Diego, CA: Academic.

Hamada FN, Rosenzweig M, Kang K, Pulver SR, Ghezzi A, Jegla TJ, Garrity PA (2008) An internal thermal sensor controlling temperature preference in Drosophila. Nature 454:217-220. CrossRef Medline

Han KA, Millar NS, Grotewiel MS, Davis RL (1996) DAMB, a novel dopamine receptor expressed specifically in Drosophila mushroom bodies. Neuron 16:1127-1135. CrossRef Medline

Hearn MG, Ren Y, McBride EW, Reveillaud I, Beinborn M, Kopin AS (2002) A Drosophila dopamine 2-like receptor: molecular characterization and identification of multiple alternatively spliced variants. Proc Natl Acad Sci U S A 99:14554-14559. CrossRef Medline

Inagaki HK, Ben-Tabou de-Leon S, Wong AM, Jagadish S, Ishimoto H, Barnea G, Kitamoto T, Axel R, Anderson DJ (2012) Visualizing neuromodulation in vivo: TANGO-mapping of dopamine signaling reveals appetite control of sugar sensing. Cell 148:583-595. CrossRef Medline

Joseph RM, Heberlein U (2012) Tissue-specific activation of a single gustatory receptor produces opposing behavioral responses in Drosophila. Genetics 192:521-532. CrossRef Medline

Joseph RM, Devineni AV, King IF, Heberlein U (2009) Oviposition preference for and positional avoidance of acetic acid provide a model for competing behavioral drives in Drosophila. Proc Natl Acad Sci U S A 106:11352-11357. CrossRef Medline

Keleman K, Vrontou E, Krüttner S, Yu JY, Kurtovic-Kozaric A, Dickson BJ (2012) Dopamine neurons modulate pheromone responses in Drosophila courtship learning. Nature 489:145-149. CrossRef Medline

Kim YC, Lee HG, Han KA (2007) D1 dopamine receptor dDA1 is required in the mushroom body neurons for aversive and appetitive learning in Drosophila. J Neurosci 27:7640-7647. CrossRef Medline

Kitamoto T (2001) Conditional modification of behavior in Drosophila by targeted expression of a temperature-sensitive shibire allele in defined neurons. J Neurobiol 47:81-92. CrossRef Medline

Kong EC, Woo K, Li H, Lebestky T, Mayer N, Sniffen MR, Heberlein U, 
Bainton RJ, Hirsh J, Wolf FW (2010) A pair of dopamine neurons target the D1-like dopamine receptor DopR in the central complex to promote ethanol-stimulated locomotion in Drosophila. PLoS One 5:e9954. CrossRef Medline

Lai SL, Lee T (2006) Genetic mosaic with dual binary transcriptional systems in Drosophila. Nat Neurosci 9:703-709. CrossRef Medline

Lin AC, Bygrave AM, de Calignon A, Lee T, Miesenböck G (2014) Sparse, decorrelated odor coding in the mushroom body enhances learned odor discrimination. Nat Neurosci 17:559-568. CrossRef Medline

Liu C, Plaçais PY, Yamagata N, Pfeiffer BD, Aso Y, Friedrich AB, Siwanowicz I, Rubin GM, Preat T, Tanimoto H (2012) A subset of dopamine neurons signals reward for odour memory in Drosophila. Nature 488:512516. CrossRef Medline

Liu Q, Liu S, Kodama L, Driscoll MR, Wu MN (2012) Two dopaminergic neurons signal to the dorsal fan-shaped body to promote wakefulness in Drosophila. Curr Biol 22:2114-2123. CrossRef Medline

Liu T, Dartevelle L, Yuan C, Wei H, Wang Y, Ferveur JF, Guo A (2008) Increased dopamine level enhances male-male courtship in Drosophila. J Neurosci 28:5539-5546. CrossRef Medline

Mao Z, Davis RL (2009) Eight different types of dopaminergic neurons innervate the Drosophila mushroom body neuropil: anatomical and physiological heterogeneity. Front Neural Circuits 3:5. CrossRef Medline

Marella S, Mann K, Scott K (2012) Dopaminergic modulation of sucrose acceptance behavior in Drosophila. Neuron 73:941-950. CrossRef Medline

Padoa-Schioppa C (2011) Neurobiology of economic choice: a good-based model. Annu Rev Neurosci 34:333-359. CrossRef Medline

Padoa-Schioppa C (2013) Neuronal origins of choice variability in economic decisions. Neuron 80:1322-1336. CrossRef Medline
Padoa-Schioppa C, Assad JA (2006) Neurons in the orbitofrontal cortex encode economic value. Nature 441:223-226. CrossRef Medline

Padoa-Schioppa C, Assad JA (2008) The representation of economic value in the orbitofrontal cortex is invariant for changes of menu. Nat Neurosci 11:95-102. CrossRef Medline

Schwartz NU, Zhong L, Bellemer A, Tracey WD (2012) Egg laying decisions in Drosophila are consistent with foraging costs of larval progeny. PLoS One 7:e37910. CrossRef Medline

Thibault ST, Singer MA, Miyazaki WY, Milash B, Dompe NA, Singh CM, Buchholz R, Demsky M, Fawcett R, Francis-Lang HL, Ryner L, Cheung LM, Chong A, Erickson C, Fisher WW, Greer K, Hartouni SR, Howie E, Jakkula L, Joo D, et al. (2004) A complementary transposon tool kit for Drosophila melanogaster using P and piggyBac. Nat Genet 36:283-287. CrossRef Medline

Tremblay L, Schultz W (1999) Relative reward preference in primate orbitofrontal cortex. Nature 398:704-708. CrossRef Medline

Venken KJ, Simpson JH, Bellen HJ (2011) Genetic manipulation of genes and cells in the nervous system of the fruit fly. Neuron 72:202-230. CrossRef Medline

Wallis JD (2007) Orbitofrontal cortex and its contribution to decisionmaking. Annu Rev Neurosci 30:31-56. CrossRef Medline

Yang CH, Belawat P, Hafen E, Jan LY, Jan YN (2008) Drosophila egg-laying site selection as a system to study simple decision-making processes. Science 319:1679-1683. CrossRef Medline

Zhang K, Guo JZ, Peng Y, Xi W, Guo A (2007) Dopamine-mushroom body circuit regulates saliency-based decision-making in Drosophila. Science 316:1901-1904. CrossRef Medline 